\title{
Les scénarios du démoniaque dans la LXX
}

Comme déjà mentionné, toute recherche sur les représentations des démons dans la LXX ne peut et ne doit pas être exclusivement réduite à une étude du vocabulaire employé pour définir les entités démoniaques. Toutefois, une ana-

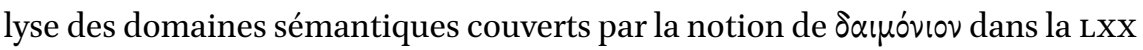
est incontournable et s'avère utile comme travail préliminaire. En effet, cette analyse permet d'une part, de déterminer les scénarios de base dans lesquels le démoniaque s'inscrit dans la LXX et les innovations potentielles amenées par rapport à la Bible hébraïque. D’autre part, une telle enquête amène à confronter la manière dont le terme est utilisé dans la LXX à des emplois plus généraux du mot à l'époque hellénistique, permettant ainsi de comprendre les relations de dépendance, écart et innovation par rapport à la catégorie de $\delta \alpha i \mu \omega v /$

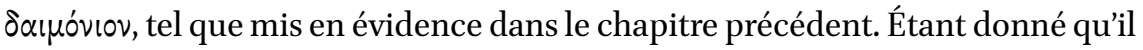
a parfois été soutenu que l'entrée de ce mot dans les textes bibliques ainsi que dans la littérature du Second Temple rédigée en grec pourrait s'accompagner d'une nouvelle conception du démoniaque ${ }^{1}$, il me semble, alors, qu'une telle investigation s'impose. Je présenterai donc les contextes où le mot $\delta \alpha \mu$ óviov fait son apparition, en les analysant à la lumière du cadre religieux et culturel hellénistique dans lequel cette catégorie s'inscrit.

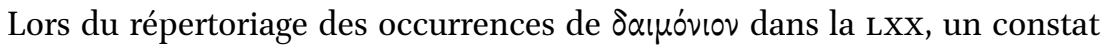
assez frappant émerge : tout d'abord, au regard du texte hébreu sous-jacent, ce terme intervient dans des contextes dans lesquels l'on ne s'attendrait pas nécessairement à le trouver. En revanche, certaines puissances dont la nature démoniaque est assez prononcée dans la Bible hébraïque ne semblent pas avoir été comprises comme des démons par les traducteurs anciens. Dans ce deuxième cas, il s'avère que ces puissances ont fait l'objet d'un procédé de re-sémantisation assez complexe rendant difficile l'identification de leur nature. Il s'agit, notamment, des deux grands démons de la tradition juive, Azazel et Lilith, mais également d'un passage obscur de la Genèse qui, d'après certaines interprétations, préserverait en hébreu un ancien nom de démon (le prétendu « démon de la porte » en Gen 4,7). Pour mieux saisir l'étendue et les limites de la notion de démon dans la LXX, il convient de nous pencher sur ces processus de transformation, en commençant par l'étude de la présence du mot $\delta a$ uóviov dans ce corpus.

$1 \quad$ Riley 1999, p. 238.

(C) ANNA ANGELINI, 2021 | DOI:10.1163/9789004468474_005

This is an open access chapter distributed under the terms of the CC BY-NC-ND 4.o licensenge Ange lini - 9789004468474 


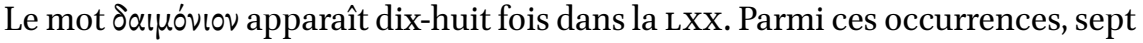
se trouvent dans des livres traduits de l'hébreu et trois dans des livres préservés ou composés seulement en grec, ou dont l'existence d'un original hébreu est discutée. En outre, sept occurrences du mot sont concentrées dans le livre de Tobit, seul contexte de prose puisque tous les autres passages sont des textes poétiques. La distribution est assez inégale : mise à part une occurrence dans le Pentateuque, le mot est attesté surtout dans les Psaumes et il paraît particulièrement apprécié par le traducteur d'Isaïe. Une vue d'ensemble des équivalences montre que, comme c'est souvent le cas dans la LXX, le terme grec traduit plus qu'un mot en hébreu. Vice-versa, le même terme hébreu peut être rendu en grec par plusieurs équivalents selon les différents contextes. Il est toutefois possible d'établir une hiérarchie des correspondances.

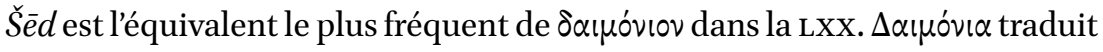
la forme plurielle šédîm une fois dans le Pentateuque, à savoir dans ce qu'on appelle couramment le «chant de Moïse », dans un passage où l'on reproche aux Israélites de vénérer de nouveaux dieux à la place de Yhwh (Deut 32,17). Cette équivalence apparait également dans le Ps 106,37 (= 105,37 LXX), dépendant de Deut 32. Le passage de Bar 4,7, dont on a seulement la version grecque, montre également une dépendance littéraire à Deut 32. On peut donc vraisemblablement imaginer que, si une Vorlage hébraïque de Baruch a existé ${ }^{2}$,

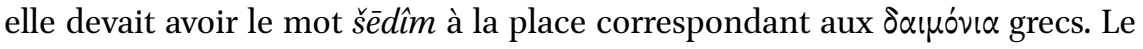
texte de Deut 32 est également reproduit dans la deuxième ode ( $\mathrm{Od} 2,17$ ), sans variations significatives pour ce verset. On notera, au passage, que tous les textes où le mot šêd apparaît datent, au plus tôt, de l'époque postexilique ${ }^{3}$. Les chercheurs ont parfois insisté sur le fait que dans la Bible hébraïque le mot est connu seulement à la forme plurielle. Il faut, toutefois, mentionner que

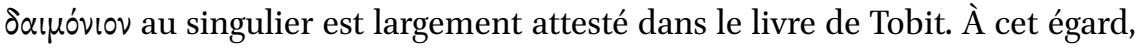
il convient de souligner que dans les manuscrits de Tobit retrouvés à Qumrân où le passage a survécu nous trouvons régulièrement les formes šd ou šd' à l'endroit correspondant ${ }^{4}$. En outre, comme nous le verrons par la suite, le $\delta \alpha \mu o^{v} \iota_{\text {ov }}$

2 L'existence d'une Vorlage hébraïque, traditionnellement acceptée par la plupart des chercheurs depuis les études de Kneucker 1879 et Tov 1975, est remise en question par la recherche récente : pour une introduction au problème, voir Assan-Dhôte et Moatti-Fine 2005, p. 55-56 et 69-71; Ryan 2015, p. 487-499; pour un modèle patchwork qui combine traduction de l'hébreu et composition en grec, voir Bogaert 2016, p. 596-597.

3 Sur la datation de Deut 32, voir infra, p. 186-187.

4 4Q196, fr. 14 i 5 ; 4Q197 frg. 4 i 13 , ii 9 et 13. 
du Ps 9o,6 $(=\text { 91,6 TM })^{5}$ correspond très probablement à la lecture d'une forme singulière wəšêd au lieu de yāšûd. Cette lecture est vraisemblablement à attribuer au traducteur, mais elle témoigne également de la familiarité des traducteurs grecs avec la forme hébraïque au singulier. L'hébreu šêd est normalement considéré comme un emprunt à l'accadien šedu ${ }^{6}$ qui désigne autant un esprit protecteur qu'un démon néfaste lorsqu'il est accompagné par le qualificatif «mauvais $»^{7}$. Bien que d'autres hypothèses aient été proposées ${ }^{8}$, cette dérivation reste la plus vraisemblable. On recense une quinzaine d'attestations du mot šéd dans les textes hébreux et araméens de Qumrân. On le trouve alors avec la graphie šdym ou šd'ym, qui signifie « démon » ${ }^{9}$. Il apparaît fréquemment en judéo-araméen et en syriaque, aux formes šyd, šyd', šydh et šydt', surtout dans les listes de démons et mauvais esprits inscrites sur les amulettes et les bols incantatoires de l'Antiquité tardive ${ }^{10}$. Il est également attesté dans les Targums ${ }^{11}$ et il deviendra le substantif générique pour «démon» en hébreu rabbinique et moderne ${ }^{12}$. Plusieurs hypothèses, que j'analyserai en détail par la suite ${ }^{13}$, ont été avancées pour comprendre l'origine et la signification des šédîm en Deut 32. L'une d'elles concerne la relation entre un dieu Shed attesté ailleurs dans le Levant et le nom divin Shadday. Toutefois, il convient d'anticiper le fait qu'en raison, entre autres, de la datation tardive du texte de Deut 32, šédîm doive ici être compris dans le sens de «démon», à savoir dans le même sens que celui attesté à Qumrân et dans la littérature du Second Temple. Il paraît, par contre, improbable que ce terme puisse garder les «restes» d'un ancien nom divin Shadday dont la signification était probablement déjà mal comprise pour les rédacteurs du Pentateuque.

$5 \quad$ Weeks, Gathercole, et Stuckenbruck 2004, p. 118, 185, 195, 197, 199.

6 Zimmern 1917, p. 69 ; AHw, p. 1208; HALOT, s.v. ; Mankowski 2000, p. 138.

7 Voir les exemples en CAD 17/2, s.v., p. 257-259; Marti 2017, p. 46-48; voir également supra, p. 37 .

8 Pour une discussion plus détaillée, voir infra, § 6.1.3.

94 4Q510 fr. 1, l. 5-8; 4Q511 fr. 10, l. 1-5 (Baillet 1982, DJD 7, p. 215-227), 11Q11 (García Martínez, Tigchelaar et Van der Woude 1998, DJD 23, p. 181-206); 4Q196, 14; 4Q197, 4 (Broshi et al. 1995, DJD 19, p. 1-56). Les occurrences ont été répertoriées et discutées par Stuckenbruck 2014 a, p. $85^{-87}$.

10 Naveh et Shaked 1998: bols no. $1 ; 2,8 ; 5 ; 10 ; 13$; amulettes no. 7 ; 7 b ; 13. Kotansky, Naveh et Shaked 1992, l. 11, 13, 17, p. 8-9 (SEG 42, 1582). La Peshitta lit šydh en Lev 17,7 à la place de s'yrm du TM : voir ch. 5 .

11 Par exemple Targum Onqelos en Lev 17,7 ; Targum Onqelos et Neofiti en Deut 32,17 ; Targum Pseudo-Jonathan en Deut 32,10 ; Targum Jonathan en Is 13,21.

12 Jastrow 1903, p. 1523-1524; Even Shoshan 1984, s.v.

13 Voir infra, p. 184-202. 
$\Delta \alpha \iota \delta^{\prime} \vee \alpha$ apparait donc comme un équivalent approprié pour šêîm, dans un contexte où la polémique contre les dieux des autres peuples revient à les comparer à des démons. Ce contexte représente l'un des scénarios majeurs d'emploi du mot dans la LXX. La polémique contre les dieux des autres est

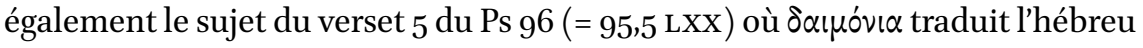
'élilìm dans l'expression : kî kol 'ělōhê hä'ammîm 'êlilîm (« car tous les dieux des peuples sont des nullités »). 'Élilìm signifie littéralement « vanités », « nullités » et est l'un des termes récurrents dans la polémique contre les images et les idoles. Ainsi, pour le traducteur grec, la polémique contre les démons et celle contre les idoles se mêlent en un seul discours.

La condamnation de pratiques cultuelles non alignées avec les prescriptions de Yhwh est également au centre du chapitre 65 du livre d'Isaïe où, au verset 3 du texte hébreu, il est question de sacrifices faits dans les jardins et d'encens brûlés sur des briques. Ici, le texte grec ajoute une expression qui désigne les

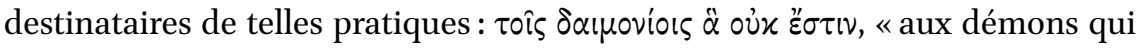
n'existent pas ». Cette expression est ponctuée d'un obèle chez Jérôme, indiquant bien qu'elle ne se trouvait pas dans l'hébreu. Une variante préservée

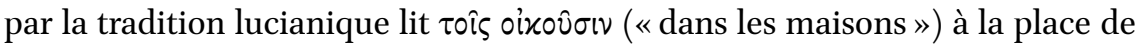
$\ddot{\alpha}$ oủx है $\sigma \tau \imath$. Cette expression pourrait traduire le premier mot du verset suivant: hayyōšabîm, lequel, sinon, n'apparait pas traduit en grec ${ }^{14}$. En tout cas,

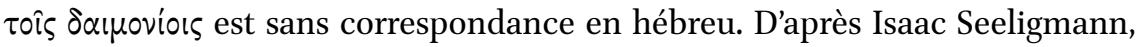

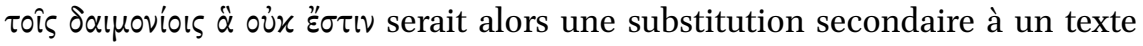

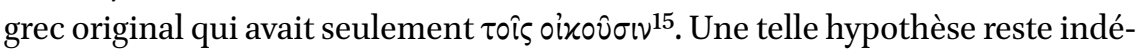

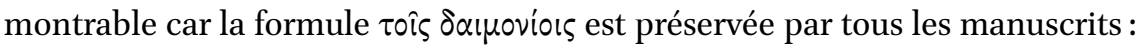
il est donc plus prudent de considérer qu'elle appartient au texte grec ancien d'Isaïe et que l'expansion exégétique remonte au traducteur ${ }^{16}$.

Un cas de figure particulier à l'intérieur de ce scénario est représenté par la LXX d'Is 65,11. Ici, on polémique contre ceux qui préparent la table pour Gad $(g d)$ et versent du vin pour Méni. Gad est attesté en hébreu biblique comme nom propre, comme toponyme et, ici, comme nom divin ${ }^{17}$. En outre, la forme $g d$ ' est bien attestée en judéo-araméen, nabatéen et palmyrénien pour indi-

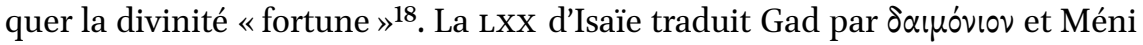
par $\tau \dot{x} \chi \eta$, «Fortune». Il faut ici relever une certaine variation dans la tradition manuscrite. Les formes $\delta \alpha \mu o ́ v i o v$ et $\tau \dot{\chi} \chi \eta$ sont inversées dans une série de

\footnotetext{
14 Ziegler 1939, p. 36o, apparatus I.

15 Seeligmann 1948, p. 31.

16 Ainsi Baer 2001, p. 176-177 ; Van der Vorm-Croughs 2014, p. 368-369.

17 Le nom de Gad fils de Leah est rattaché par étymologie populaire au bon sort (Gen 30,11), voir infra, § 6.2.2. Le toponyme Ba'al-Gad apparaît en Josué 11,$17 ; 12,7 ; 13,5$. 


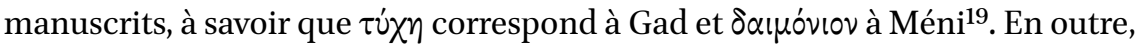
le Vaticanus et l'Alexandrinus préservent le datif $\delta \alpha \mu o v i \omega$ alors que le Sinaiticus et d'autres témoins du groupe alexandrin ont la forme $\delta$ ápovi, dérivée de $\delta \alpha i \mu \omega \nu^{20}$. Il est très probable, comme le suggéraient, déjà, Isaac Seeligmann et, plus récemment, Joachim Schaper ${ }^{21}$, que le traducteur ait reconnu dans les sacrifices offerts à Gad et Méni des formes de culte à la bonne fortune et au bon démon (désigné sous le terme d'agathodaimon), extrêmement populaires aux époques hellénistique et romaine, en Égypte et dans le Levant. Cette interprétation pourrait également justifier la présence de la forme $\delta \alpha i \mu \omega \nu$ comme forme originale choisie ici par le traducteur. Ainsi, dans ce cas spécifique, et tout en restant dans le contexte de la polémique contre les dieux étrangers, les divinités ne sont pas «réduites» à des démons comme dans les autres passages ${ }^{22}$, mais font directement référence aux cultes hellénistiques du bon démon et de la bonne fortune.

Un autre contexte où le $\delta \alpha u$ óviov apparaît est celui des maladies et des attaques démoniaques proprement dites. Ce scénario est notamment décrit dans le Ps 91 (= 9o LXx) où le traducteur lit, comme nous l'avons vu, wəšéd («et le démon») au lieu du verbe yāšûd (dérivé de šdd, « détruire », « ravager»). Dans le texte hébreu, d'autres agents divins, tels que Qețeb et Deber, apparaissent comme responsables de maux pouvant frapper les hommes. De la même manière dans la LXX, la représentation des démons comme agents de

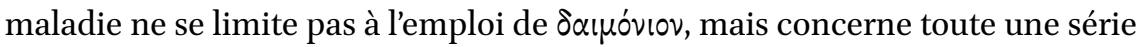
d'autres figures et agents dont la fonction doit être analysée au cas par cas ${ }^{23}$. Les attaques démoniaques sont également l'un des noyaux narratifs du livre de

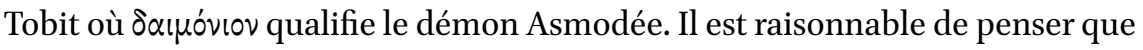
les attaques démoniaques dans le Ps 91 et dans le livre de Tobit ne soient pas envisagées comme maladie générique, mais plus spécifiquement comme une forme de possession. En tant qu'activité attribuée par excellence aux démons et étant bien attestée dans la Bible hébraïque, la possession représente donc un scénario ultérieur pour l'exploration de l'action du démoniaque. Comme Daniel Barbu l'a bien relevé24, il faut toutefois préciser que dans la Bible ce type

\footnotetext{
19 Voir Ziegler 1939, p. 361, apparatus I. La correspondance $\tau \dot{x} \chi \eta$-Gad est également rétablie par les réviseurs (ibid., apparatus II).

20 Voir Ziegler 1939, p. 361, apparatus I. Cette forme est d'ailleurs préférée ici par Rahlfs.

21 Seeligmann 1948, p. 99-100; Schaper 2010.

$22 \quad$ Pace Riley 1999, p. 238.

23 Voir infra, ch. 4.

24 Barbu et Rendu-Loisel 2009, p. 307-308. Voir infra, ch. 7.
} 
d'activité est confié davantage à des entités qualifiées comme « esprits » (rûhôt , qui correspondent toujours au grec $\pi v \varepsilon \dot{\mu} \mu \alpha \tau \alpha)$ et, plus rarement, aux $\delta \alpha \iota \operatorname{ó}^{2} \alpha^{25}$.

Le terme $\delta \alpha \mu o ́ v i \alpha$, toujours au pluriel, a d'autres équivalents, notamment dans la LXX d'Isaïe où il traduit le mot śs'îrîm, littéralement «bouc», en Is 13,21, et peut-être l'hébreu șiyyîm en Is 34,14, un terme à la compréhension difficile qui renvoie aux habitants du désert et de la steppe. Nous nous trouvons, en effet, dans un contexte complètement différent: celui d'habitants de ruines, à savoir de villes qui ont été détruites et abandonnées à jamais, et dont la Babylone d'Is 13 est le paradigme. Dans un contexte similaire, on peut relever l'occurrence $\delta \alpha \mu o ́ v i \alpha$ de Bar 4,35, également en référence à Babylone. Le choix

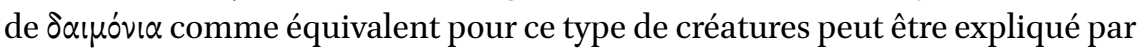
l'approche exégétique par laquelle le traducteur du livre d'Isaïe a compris et retravaillé le paysage culturel et religieux lié à l'espace des ruines. Cet espace devra également être analysé en détail ${ }^{26}$.

Essayons, à présent, de récapituler ces quelques observations sur l'usage de

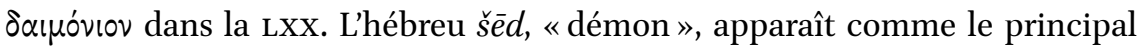
équivalent pour le grec $\delta \alpha u o ́ v i o v:$ celui-ci traduit šêd trois fois sur sept; šêd

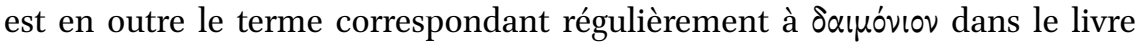
de Tobit. Il faut alors comprendre pourquoi différents traducteurs ont choisi

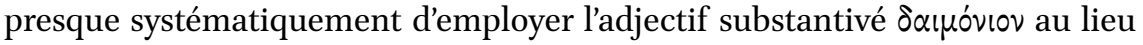
du substantif plus commun $\delta \alpha i \mu \omega \nu$ qu'ils avaient à disposition. Le choix des traducteurs s'inscrit dans un usage du mot de plus en plus spécialisé et bien

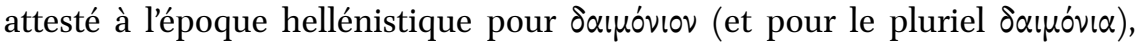
pour indiquer des entités malfaisantes et différenciées des dieux. En revanche, $\delta \alpha i \mu \omega \nu$ est une notion religieuse qui reste beaucoup plus complexe et fortement intrinsèque à la religion grecque. En outre, son lien étroit avec la notion de destin rendait son usage difficile dans les contextes que l'on vient de décrire. Par rapport à $\delta \alpha i ́ \mu \omega \nu, \delta \alpha \mu o ́ v i o v$ a un domaine sémantique qui est à la fois plus spécialisé et moins connoté, surtout à l'époque des traducteurs. De ce point de vue, ce n'est pas un hasard si la seule exception où la tradition manuscrite préserve $\delta \alpha i \mu \omega \nu$ est en Is 65,11 , passage qui fait vraisemblablement référence au culte grec du bon démon. En outre, il faut considérer que l'équivalence entre

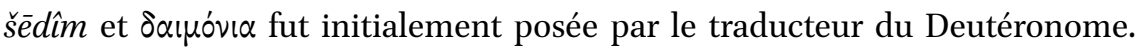
Par conséquent, elle a été gardée telle quelle dans une série de passages qui montrent une dépendance littéraire ou thématique à l'égard de Deut 32. En choisissant cette équivalence, les traducteurs contribuent à réduire l'ambiguïté

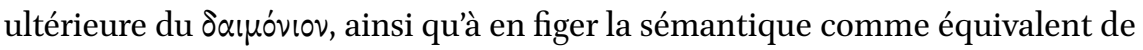

25 Voir, par exemple, Jud 9,23; 1 S 16, 1-13; 1 R 22, 17-25 ; 2 Ch 18, 22.

26 Voir infra, ch. 5 . 
« démon». Ce processus sera prolongé et complété dans d'autres traditions grecques du Second Temple mais aussi plus tard. Les versions grecques d'Hénoch, par exemple, utilisent encore $\delta \alpha \mu o ́ v i \alpha$ pour parler des sacrifices aux démons faits par les humains détournés par les anges veilleurs, lesquels se sont unis aux femmes ${ }^{27}$. Dans la version grecque du livre des Jubilés, Mastema est

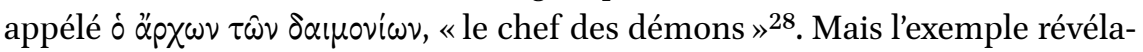
teur en ce sens est, sans conteste, le Nouveau Testament qui enregistre environ soixante occurrences de $\delta \alpha \mu o ́ v i o v$ alors que $\delta \alpha i \mu \omega \nu$ n'y apparaît qu'une seule fois ${ }^{29}$.

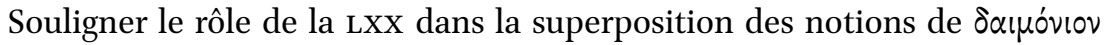
et de « démon» ne signifie cependant pas nécessairement que la sémantique

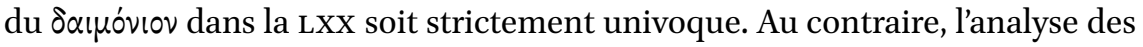
contextes dans lesquels le mot apparaît montre une sémantique encore «plurielle », et ce, à bien des égards. Une telle pluralité est également démontrée par le fait que d'autres équivalences sont possibles: outre celle entre $\delta \alpha \mu \delta^{\prime} v \alpha$ et šédîm, on retrouve une correspondance entre $\delta \alpha \mu o ́ v i \alpha$ et «idoles » ou entre $\delta \alpha \_\mu o ́ v i \alpha$ et certaines créatures qui peuplent les ruines. De plus, la manière

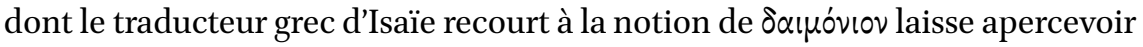
des différences significatives par rapport aux usages de cette catégorie dans le reste de la Lxx. Dans d'autres traditions du judaïsme hellénistique, notamment chez Philon et chez Flavius Josèphe, on repère d'ailleurs des occurrences de $\delta \alpha i \mu \omega \nu$ et $\delta \alpha \mu o^{\prime} v i o v$ encore parfaitement alignés aux usages philosophiques et littéraires classiques, à savoir que ces termes sont employés avec la signification de «destin », « divinité inconnue », voire tout simplement « divinité », et même d'« esprit défunt ${ }^{30}$. Ces exemples nous montrent que la littérature

271 Hén 19,1-2 ; 99,7. Voir A.T. Wright 2005 b, p. 155-156 ; D.B. Martin 2010, p. 666-667.

28 Jub 17,16. Dans cet épisode, qui raconte le sacrifice d'Isaac, le nom de Mastema remplace

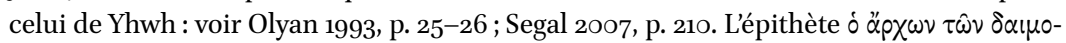

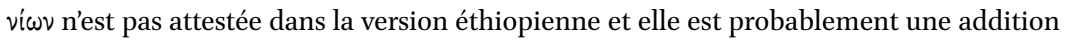
tardive, voir Charles 1917, p. 252. Sur ce passage voir également Brand 2013, p. 182-185.

29 Mt 8,31. $\Delta \alpha i \mu \omega \nu$ apparaît également dans le textus receptus de Mc 5,12; Luc 8,29; Ap 16,14 ; 18,2 : il s'agit, en tout cas, d'un nombre très réduit d'occurrences.

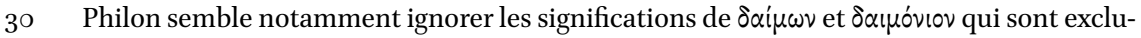
sives à la LXX. Il utilise souvent $\delta \alpha i ́ \mu \omega \nu$ comme synonyme de $\theta$ rós pour indiquer une entité surnaturelle générique (outre Virt. 172, où il cite Pindare, on peut voir Legat. 112 et Hypoth. 6, 9), y compris dans un sens proche de celui de «sort», « destinée «(voir surtout Flacc. 168, mais également Flacc. 179, Prob. 130, Prov. 2, 8). On peut voir également l'expression $\varkappa \alpha \tau \dot{\alpha} \delta \alpha \dot{\prime} \mu o v \alpha$, «sous inspiration divine » ou simplement «de manière providentielle »

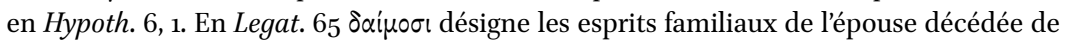
Caligula, notamment dans leur fonction d'esprits vengeurs alertés par un meurtre à l'intérieur de la famille (celui de Silanus, beau-père de l'empereur et tué par ce dernier), ce

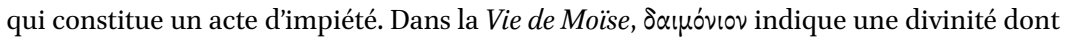


juive hellénistique ne constitue pas une exception par rapport à la polysémie du $\delta \alpha i \mu \omega \nu$ dans le reste de la littérature grecque.

Enfin, l'enquête sur la distribution du terme $\delta$ aluóviov dans la LXX a permis de dégager une pluralité de scénarios ou de domaines dans lesquels les démons interviennent et qui seront analysés chacun en détail dans les chapitres suivants: celui des agents divins responsables de maladies et des fléaux; l'espace des habitants des ruines et des villes abandonnées ; la représentation des dieux des autres et la relation entre démons et idoles. Ces scénarios sont présents à la fois dans la Bible hébraïque et dans la LXx, mais leurs déclinaisons et différences n'ont pas encore suffisamment été mises en valeur. En outre, d'autres notions «clés » de la représentation du démoniaque dans la Bible hébraïque émergent dans ce cadre. Elles sont surtout liées à l'activité des esprits : puissances agentielles de la divinité et premières responsables des formes de possession, et dont l'analyse doit nécessairement être prise en compte lors d'une enquête sur les représentations du démoniaque dans la Bible. Avant d'analyser singulièrement chacun de ces contextes, il faut, toutefois, encore s'interroger sur la prétendue disparition dans la LXX de certains démons «majeurs » de la Bible hébraïque.

\section{Le va-et-vient des démons : Azazel et Lilith}

\subsection{Azazel dans la Bible hébräque}

Protagoniste du grand rituel annuel de purification de la communauté décrit en Lévitique 16, Azazel est à bien des égards la principale figure démoniaque de la Bible hébraïque. Il n'est jamais nommé en dehors de ce chapitre qui est, par ailleurs, assez pauvre en détails concernant ses origines, son aspect ainsi que son histoire. Toutefois, le rôle qu'il joue à l'intérieur du rituel permet d'avancer un certain nombre d'éléments concernant sa fonction et son rapport à Yhwh. Tout d'abord, Azazel est présenté comme une entité clairement distinguée de Yhwh, et qui, comme le démontre la structure même du rite composé de plusieurs sacrifices, lui est soit mise en parallèle, soit en opposition. Le tirage aux sorts des boucs ouvre la partie centrale du rituel de purification qui s'étend

on ne connaît pas le nom (Mos. 1.276: Balaam et Balaq s'arrêtent sur une colline où il $\mathrm{y}$

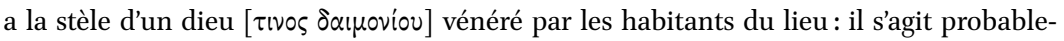

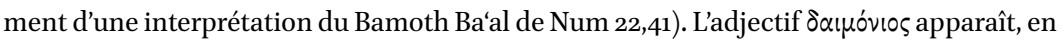
outre, deux fois dans le sens de «suprahumain», « extraordinaire» (Aet. 47, et 64). Pour le rapport entre anges et démons chez Philon voir infra, p. 204. Pour un traitement plus détaillé des continuités et des discontinuités par rapport à la notion de « démon » dans la LXX chez les auteurs juifs hellénistiques on peut voir Angelini, à paraître. 
du verset 7 au verset $22^{31}$. Dans un premier temps, alors qu'un des boucs est sacrifié pour Yhwh $(l y h w h)$, l'autre est envoyé, vivant, à Azazel dans le désert (la'ăzā'zél hammidbārâ, versets 8-10). Deuxièmement, le verset 9 souligne que le bouc destiné à Azazel doit être présenté au préalable devant Yhwh: ce geste semble indiquer une forme d'autorité de Yhwh sur Azazel. Ces deux puissances sont donc mises en parallèle dans le rituel sans avoir, pour autant, un pouvoir équivalent. Troisièmement, le texte souligne le fait qu'Azazel est une puissance strictement associée au désert et aux espaces chaotiques. En effet, au verset 10, le bouc pour Azazel est envoyé dans le désert, ceci étant mentionné par son nom courant midbār. Au verset 22, qui explique la signification du rite pour Azazel (à savoir le fait que le bouc emporte avec lui les fautes de la commu-

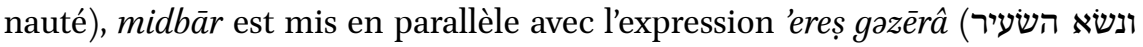
et le bouc emportera sur lui toutes les fautes vers une terre stérile, et il enverra ce bouc dans le désert»). Cet adjectif attribué au sol est généralement traduit par « infertile », « stérile », en se fondant sur sa signification en arabe. Littéralement, l'expression signifie plus précisément «terre coupée », «terre séparée», puisque gazērâ dérive de la racine $g z r$, «couper». Elle indique donc, non seulement, son éloignement de l'espace humain, mais également du domaine d'influence de Yhwh, à savoir le territoire où s'exercent les relations entre les hommes et la divinité. Comme le montre le Ps 88,6 ce territoire peut, en outre, être métaphoriquement comparé au monde des morts : les défunts sont « coupés » de la présence divine avec laquelle ils perdent contact: במתים חפשי כמו חללים שכבי קבר אשר (abandonné parmi les morts, comme les 'percés' couchés dans la tombe, et dont tu as perdu le souvenir car ils sont coupés de ta présence ») $)^{32}$. Le sens de l'expression 'ereș gazērâ en Lev 16,22 était encore clair pour le traducteur grec, qui rend gazērâ par l'équivalent inhabituel $\alpha^{\prime} \beta \alpha-$ $\tau \circ \varsigma$, « inaccessible», adjectif parfaitement approprié à ce contexte ${ }^{33}$.

Enfin, il importe de relever qu'à l'intérieur de la construction rituelle complexe de Lev 16, Azazel n'est pas le destinataire d'un sacrifice à proprement parler, ni d'une offrande, mais plutôt d'un rite d'élimination qui a pour but d'emporter les fautes de la communauté, lesquelles sont transférées sur le bouc, comme l'expliquent les versets 21 à 22 . Le fait que ce bouc reste vivant, ce

31 Pour une analyse détaillée du rite de purification annuelle de Lev 16, qui est en réalité beaucoup plus long et complexe, et composé de différents sacrifices, on peut voir Milgrom 1991, p. 1009-1084; plus récemment, Nihan 2007, p. 340-371 et la bibliographie relative.

32 Ps 88,6. Sur l'expression 'ereș gazērâ, voir les remarques de Tawil 1980, p. 55-56; Nihan 2007, p. 354-355.

Voir, à ce sujet, les remarques de Pietersma 2006 b, p. 410-412. 
qui est rappelé à plusieurs reprises dans le texte, met en évidence la différence entre le rite destiné à Azazel et le sacrifice destiné à Yhwh ${ }^{34}$. Les parallèles proche-orientaux et, plus largement, méditerranéens à ce rituel d'élimination ont été largement étudiés et ne doivent pas être rappelés ici ${ }^{35}$. Il importe toutefois de souligner que le rituel pour Azazel se différencie de la procédure sacrificielle normale. En effet, celle-ci implique une forme de négociation entre les deux parties. À l'inverse, le rituel de purification vise à écarter, éliminer, voire faire disparaître à jamais les fautes de la communauté : comme tel, il se rapproche des rituels typiquement adressés aux démons dans l'Antiquité36.

Les traits associés à Azazel tel qu'il est décrit en Lev 16 - c'est-à-dire l'appartenance à la sphère du chaos, la subordination à Yhwh et le fait d'être destinataire d'une pratique de détournement - appartiennent pleinement au « réseau » qui caractérise le démoniaque au Levant, tel que je l'ai défini dans le premier chapitre. Ainsi, il est apparu difficile, même pour ceux qui ont cherché à expulser toute trace du démoniaque de la Bible hébraïque, de ne pas reconnaître en Azazel une figure de démon ${ }^{37}$.

Une interprétation séduisante qui mérite d'être discutée plus en détail est celle récemment avancée par Aron Pinker, pour qui Azazel serait une forme ou une manifestation de Yhwh dans le désert. Pour comprendre cette hypothèse il faut revenir sur la question de l'étymologie du nom d'Azazel. Sur le plan philologique, l'hypothèse la plus robuste et la plus largement acceptée est celle proposée par Bernd Janowski et Gernot Wilhelm. Ils considèrent le nom Azazel comme composé de la racine du sémitique commun 'zz (en accadien Azazu/ $e z e z u$, « être en colère »; en hébreu et dans les autres langues ouest-sémitiques la racine signifie d'habitude « être fort », «être fier ») suivi par le nom divin ' 138 .

\footnotetext{
34 Lev 16,10 et $20-22$.

35 Les études classiques sont Wright 1987, p. 15-74 ; Janowski et Wilhelm 1993 ; pour le dossier comparatiste grec un bon point de départ est Bremmer 1983 .

36 Voir supra, p. 25-40; p. 53-59. Voir, en ce sens, les parallèles avec les rituels contre les démons analysés par D. Wright 1987, p. 65-69.

37 Blair (2009, p. 62) considère Azazel comme le nom propre d'un être surnaturel qui réside dans le désert, personnification du chaos et opposé à Yhwh : autrement dit, elle mentionne les principaux traits qui caractérisent un démon au Levant. En revanche, l'analyse de Frey-Anthes (2007, p. 61-63) est plus subtile. Elle a sans doute raison de rejeter la superposition immédiate de certaines créatures hybrides, telles que, par exemple, les boucs représentés sur les ivoires de Megiddo, avec l'Azazel biblique car une telle association n'est pas suffisamment appuyée par les éléments textuels dont nous disposons. Néanmoins, sa compréhension d'une figure religieuse complexe comme Azazel exclusivement comme une « literarische Gestalt» (ibid., p. 240-241) me paraît réductrice. Pour une critique du concept de paganisme littéraire, voir infra, p. 117-118.

38 Voir également Dietrich et Loretz 1993 ; pour d'autres hypothèses étymologiques, voir la discussion en Janowski 1999 a, p. 128-131.
} 
Cette lecture semble, entre autres, confirmée par la présence d'une divinité Aziz ou Azuz attestée par le théophore phénicien ' $b d^{\prime} z z$, « serviteur d'Azuz ${ }^{39}$ ainsi que par un théonyme gaditain 'zzmlk'štrt, «Le fort Milkaštart» ${ }^{40}$. Un théonyme ${ }^{d} A-z u-z i$ est, en outre, attesté en néo-assyrien ${ }^{41}$ et un nom propre avec cette racine apparaît déjà à Ebla ${ }^{42}$. Il paraît également assuré que ' $z z^{\prime} l$ est la graphie originaire du texte biblique car cette forme est préservée dans deux textes retrouvés à Qumrân (voir tableau $12 b$ en Appendice). Le premier est le Rouleau du Temple, dans un passage qui réécrit le rite de Lev $16^{43}$. Comme c'est souvent le cas dans les écrits qumrâniens, le texte biblique est reporté avec des réarrangements et de subtiles variations. Ainsi, le passage en question nous dit que le bouc est envoyé à ‘̆zaz'él hammidbārâ, une expression qui d'après William Gilders pourrait être comprise comme «Azazel du désert», presque comme une épithète d'Azazel ${ }^{44}$. Le deuxième texte est un pesher sur Azazel et les anges (4Q18o), à savoir un commentaire du texte biblique. Dans ce cas, le récit de la création est réécrit et donc réinterprété, et Azazel y est nommé deux fois avec la graphie ' $z z^{\prime} l^{45}$. Cette graphie se retrouve encore sur quelques manuscrits du Pentateuque samaritain avec une première occurrence du nom en Lev 16,1046. La métathèse entre zayin et aleph qui a donné lieu à la forme massorétique inhabituelle ' $z$ ' $z$ len Lev 16 est probablement explicable par une forme de révérence du rédacteur envers le nom divin, et ce, à un stade ultérieur de la transmission du texte, mais, néanmoins, antérieur à la traduction grecque. On rappellera à cet égard que les procédés de métathèse sont souvent employés pour des modifications phonétiques motivées par des raisons d'ordre «tabouistique ${ }^{47}$. Si la question étymologique est donc relativement claire sur le plan philologique, elle l'est moins au niveau de l'interprétation. La forme ' $z z$ ' l pourrait être comprise soit selon la signification courante de la racine sémitique occidentale, c'est-à-dire comme « la force du dieu », ou encore

39 Zadok 1978, p. 57-58.

40 Lipiński 1992, p. $5^{2}$; Krahmalkov 200o, p. 363, s.v. 'z. Voir également les théophores ' $z(z) b$ ' et 'zzmlk: Lipiński, ibid.

41 Zadok 1978, p. 57.

$42 A z i z a$, Vattioni 1987. La racine ' $z z$ est, d'ailleurs, à l'origine à une série de noms propres en hébreu biblique : 'Azaz, 'Uziel, 'Uza, etc.

43 11QTS 26, l. 13.

44 Gilders 2012, p. 69.

454 Q180, fr .1, l. 7-8. Suite à l'editio princeps d'Allegro (1968, DJD 5, p. 79-80), le texte a été réédité par Dimant 1979 (= 2014, p. 385-390 ; voir également Garcia Martinez et Tigchelaar 1997, p. 371-373). Pour une comparaison entre ce texte et les traditions hénochiques, voir Davidson 1992, p. 271-274; plus récemment, Gilders 2012.

46 Voir Schorch 2018, p. 137 et tableau $12 a$ en Appendice.

47 Mayer Modena 1982. Pour la notion de tabou linguistique, voir Cardona 1976, p. 140-141. 
«le dieu fort »; soit comme la « colère du dieu ». Cette dernière interprétation serait signifiante dans le contexte de Lev 16. De plus, elle nous présenterait la colère comme un agent de la divinité, ce qui a d'autres parallèles dans la Bible hébraïque ${ }^{48}$. Cependant, cette interprétation demeure peu probable car la signification de ' $z z$ comme « colère » n'est jamais attestée en sémitique occidental, alors que la valeur de «force» est bien présente dans toute l'histoire du sémitique du nord-ouest, à savoir de l'ougaritique au judéo-araméen ${ }^{49}$. $\mathrm{Au}$ passage, il vaut la peine d'observer qu'un papyrus grec chrétien provenant d'Égypte, contenant une liste des noms divins et ayant, peut-être, été destiné à être utilisé comme amulette, explique encore le nom grec $A \zeta \alpha \eta \lambda$ comme signifiant «la force de Dieu $»^{50}$. Dans ce contexte, Pinker comprend Azazel comme signifiant «dieu fort, fier »; il s'appuie sur l'existence du dieu Azuz dans le monde ouest-sémitique pour conclure que ce «dieu fort » qui réside dans le désert en Lev 16 ne serait autre que Yhwh lui-même. Plus précisément, il s'agirait d'une même divinité, vénérée sous le nom de Yhwh dans le temple de Jérusalem et sous le nom d'Azazel au désert ${ }^{51}$. Toutefois, à mon avis, cette hypothèse se heurte à une difficulté provenant du texte de Lev 16. Comme nous l'avons vu, au verset 10, on spécifie que le bouc destiné à Azazel doit, auparavant, être présenté vivant devant Yhwh pour l'expiation. La formulation du rituel semble donc indiquer un rapport de subordination d'Azazel à Yhwh plutôt qu'une identification entre les deux divinités. En outre, la qualification du désert comme 'ereṣ gazērâ, « terre séparée », met l'accent sur la distance et l'éloignement de la présence divine. Même si le nom d'Azazel pourrait originellement renvoyer à celui d'une divinité proche-orientale, les indices pour une identification entre Yhwh et Azazel dans le contexte du rituel de Lev 16 demeurent, à mon avis, assez faibles. Les deux sont plutôt présentés comme deux divinités distinctes, dont la deuxième a un domaine d'action plus limité et reste associée à la sphère du chaos : deux traits qui ont été définis ici comme étant compatibles avec la sphère du démoniaque. En revanche, l'on pourrait se demander si le dieu proche-oriental Azuz - si c'est bien de lui qu'il s'agit n'a pas subi un processus de «démonisation » lors de sa transposition dans la Bible hébraïque, selon un processus assez similaire à celui à l'œuvre pour d'autres divinités levantines, telles que Resheph et Deber ${ }^{52}$.

48 Voir, notamment, Num 17 et infra, § 4.1.

49 La racine peut avoir des nuances différentes : « être fort », « être insolent », « être fier ». Le domaine sémantique est celui de la force et de l'énergie. Voir S. Wagner 2001.

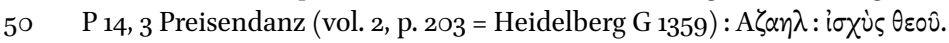

51 Pinker 2007, p. 14-16.

52 Voir infra, ch. 4. 
Si Azazel dans la Bible hébraïque ne peut être compris autrement que comme un démon, le fait qu'il ait été, pour ainsi dire, évacué de la traduction grecque soulève nécessairement des interrogations. Avant d'analyser plus en détail le texte grec et les procédés mis en œuvre par le traducteur, il faut revenir d'abord sur les problèmes liés à la transmission ancienne du nom d'Azazel et sur la diversité de la réception de cette figure dans l'Antiquité. Ces données permettront de mieux comprendre le cadre dans lequel les traditions de la LXX se situent.

\subsection{La réception ancienne d'Azazel}

Selon une dynamique assez courante dans l'exégèse ancienne, l'absence de détails sur Azazel dans la Bible hébraïque ainsi que la difficulté de son nom ont donné lieu à un processus d'expansion «midrashique» considérable dans la réception de cette figure. Tout d'abord, la nature démoniaque d'Azazel comme démon est amplifiée dans les traditions du Second Temple et captée par l'apocalyptique. Les traditions hénochiques préservées dans le Livre des Veilleurs, un fragment des livres des Géants préservé à Qumrân, ainsi que d'autres textes qumrâniens tels que le pesher 4Q18o, inscrivent Azazel parmi les anges rebelles qui se sont unis aux femmes, engendrant la race des géants. Dans le Livre des Veilleurs, un ange nommé Asaël/Azazel est également responsable d'avoir enseigné aux hommes les arts métallurgiques, la teinture et l'art de travailler les pierres précieuses. L'insertion d'Azazel à l'intérieur du mythe des Veilleurs dans le premier livre d'Hénoch est reconnue comme secondaire par rapport au noyau original du mythe ${ }^{53}$ et implique la connaissance et l'appropriation de thèmes provenant du chapitre 16 du Lévitique ${ }^{54}$. La tradition qui voit en Azazel un ange est également attestée dans les Oracles Sibyllins et dans le Testament de Salomon ${ }^{55}$; elle est particulièrement complexe dans

53 Le texte de 1 Hen $6-36$, connu également comme «Livre des Veilleurs», représente la partie la plus ancienne du livre d'Hénoch. Ici, un ange nommé Asaël ou Azazel est mentionné aux passages 6,$7 ; 8,1 ; 9,6 ; 10,4 ; 10,8 ; 13,1$ (voir aussi 1 Hen 54,5-6; 55,4; 69,2 qui ne sont pourtant attestés ni en grec ni en araméen). Le matériel narratif contenu dans le Livre des Veilleurs a une histoire littéraire complexe : la plupart des chercheurs reconnaît l'insertion d'Asaël/Azazel comme secondaire ou tertiaire. Voir Nickelsburg 2001, p. 191201, et en dernier l'analyse de Bhayro 2005. Je remercie Matteo Silvestrini pour nos discussions autour de la présence d'Azazel dans le livre d'Hénoch.

54 Voir, à ce sujet, Hanson 1977 ; Nickelsburg 1977 ; id. 2001 ; Stuckenbruck 2014 b ; Silvestrini, à paraître. Sur la réception d'Azazel dans les traditions juives anciennes, voir également Stökl Ben Ezra 2003, p. 85-95, 124-134.

Sib. Or. 2,215 ; T. Sol. 7,7 . 
l'Apocalypse d'Abraham ${ }^{56}$ et se retrouve également dans les papyri magiques de l'Égypte gréco-romaine ${ }^{57}$.

Par la suite, une ligne d'exégèse minoritaire interprète l'Azazel biblique comme faisant référence au nom du lieu où le bouc est envoyé. Le passage le plus connu se trouve dans un traité du Talmud ${ }^{58}$, mais cette interprétation est également attestée par quelques Targums ${ }^{59}$ et elle est probablement connue par Aquila.

Enfin, l'on pourrait mettre en évidence une troisième ligne d'interprétation d'origine ancienne et faisant d'Azazel le bouc émissaire. Il s'agit d'une tradition fondée essentiellement sur la traduction de la Vulgate, notamment sur le caper emissarius traduit par Jérôme ${ }^{60}$, qui n'a pas de véritable correspondant dans le texte hébreu. Cette interprétation a joui d'un certain succès dans les milieux chrétiens anciens (et modernes), et a été rapprochée, parfois improprement, des traditions grecques sur le $\varphi \alpha p \mu \alpha x o ́ \varsigma^{61}$. Il convient, à cet égard, de préciser que, contrairement à certains rituels hittites et grecs, dans le texte hébreu de Lev 16, le bouc pour Azazel ne fonctionne pas comme un véritable substitut des péchés du peuple. En effet, le texte ne contient aucune indication concernant le fait que le bouc souffre à la place du peuple ou qu'il soit offert en substitution aux péchés : il est plutôt un véhicule chargé de transporter les fautes du peuple le plus loin possible ${ }^{62}$. La LXX est parfois indiquée comme supportant l'interprétation d'un « bouc émissaire » ${ }^{63}$ : en réalité, comme on le verra, l'opération mise en œuvre par le traducteur est un peu plus complexe.

Une telle variété d'interprétations de la figure d'Azazel est générée, entre autres, par les difficultés posées par son nom. Si la forme originelle du texte biblique peut être reconstruite avec une relative certitude, le nom, quant à lui, fait l'objet d'un certain nombre de variations dès l'époque du Second Temple (voir tableau $12 a$ et $b$ en Appendice). Plusieurs graphies sont attestées parmi les manuscrits de la Mer Morte. L'orthographe ' $z$ ' $z$, à savoir la même que dans le TM, est attestée dans un fragment araméen de Qumrân du Livre des Géants. Bien que le mot ne soit pas entièrement lisible, la restitution est très

\footnotetext{
$5^{6} \quad$ Voir, à ce sujet, les analyses de Grabbe 1987 ; Fauth 1998.

$57 \quad P G M 45,7$.

58 b.Yoma 67b.

59 Targum Jonathan en Lev 16,10.

6 o Lev 16,8 , 10 et 26 .

$61 \quad$ Notamment à la suite du travail de Girard 1972.

62 Voir, à ce sujet, les remarques de D. Wright 1987, p. 49-5o.

63 Blair 2009, p. 17.
} 
vraisemblable du point de vue paléographique ${ }^{64}$. En outre, la transmission du nom est compliquée dans les traditions sur les Veilleurs qui ont été transmises en araméen et en grec avant d'être traduites en éthiopien. L'histoire d'Azazel dans le livre d'Hénoch est rattachée à la liste des Veilleurs donnée en 1 Hen 6,7, où les noms des anges veilleurs sont mentionnés pour la première fois. Là apparaît un certain Asaël/Azaël dont le nom est transcrit de différentes manières : les fragments de Qumrân, qui représentent la forme textuelle la plus ancienne, préservent les graphies 's'l('asa'el) ${ }^{65}$ et 's'l ('aśa'el) ${ }^{66}$ dans le passage correspondant à 1 Hen 6,7; les codices grecs ont, pour leur part, A $\sigma \varepsilon \alpha \lambda$ (Panoplitanus), $A \zeta \alpha \lambda \zeta \eta \lambda$ (Syncellus) ${ }^{67}$. Il est probable qu'à l'origine cet Asaël constituait une entité divine distincte d'Azazel, mais que, au cours de la tradition, du fait de la similitude phonique, ce dernier ait été rapproché du premier puis lui ait été assimilé. Quoiqu'il en soit, un fragment araméen du Livre des Géants et le pesher préservé en 4Q180 montrent que la forme «biblique » du nom (Azazel) était déjà associée au mythe des Veilleurs au moins au premier siècle avant notre ère. Au passage, notons que la forme grecque préservée dans toutes les occurrences du texte grec d'Hénoch (à l'exception de 1 Hen 6,7) est $A \zeta \alpha \eta \lambda^{68}$. Elle pourrait refléter une lecture « à la grecque » d'un original ' $z z^{\prime} l$ (le zeta étant une consonne double), les deux formes 'asa'el et 'aśa'el de 1 Hen 6,7 attendant plutôt un sigma comme équivalent du samek ou du sin. En outre, une variété de graphies grecques et araméennes est attestée sur les papyri magiques et sur les bols incantatoires car, évidemment, ce nom fonctionnait bien dans ses différentes formes en tant que nomen magicum ${ }^{69}$. La Peshitta de Lev 16 a partout la forme ' $z z^{\prime} y l$ : il se peut que l'insertion du yod vise ici également à effacer le nom divin ' $E l$, introduisant peut-être une référence au bouc ('ayil), selon une interprétation similaire à celle que l'on retrouve dans les traditions grecques et latines.

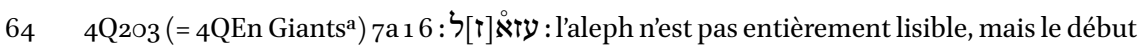
de la lettre laisse entrevoir un signe différent de celui du zayin qui précède. Voir Milik 1976 a, p. 312-314; Stuckenbruck 1997, p. 77-83 ; id. 2000 (DJD 36, p. 19-24). Une reproduction récente du fragment est disponible en ligne : https://www.deadseascrolls.org.il/ explore-the-archive/image/B-298789. Sur les raisons du lien entre Azazel et le mythe de géants, voir Stuckenbruck 1997, p. 81-82.

654 Q2O1 $\left(=4 \mathrm{QEn}^{\mathrm{a}}\right) 3,9$.

$664 \mathrm{Q}_{204}\left(=4 \mathrm{QEn}^{\mathrm{c}}\right) 2,26$.

67 Voir Charles 19o6, p. 15. Pour la discussion de la transcription du nom en éthiopien, on peut voir en dernier Silvestrini, à paraître.

681 Hen 8,$1 ; 9,6 ; 10,4,8 ; 13,1$; et Sib. Or. 2,215.

69 Toutes les occurrences ont été répertoriées par Fauth 1998. 


\subsection{Azazel dans la $L X X$}

Par rapport à ces traditions anciennes qui développent la figure d'Azazel dans de multiples directions en en intensifiant souvent ses qualités « démoniaques », la LXX semble représenter un cas de figure particulier. En effet, non seulement elle ne préserve pas le nom d'Azazel, mais, de plus, le traducteur utilise quatre solutions différentes pour l'évoquer, ce qui mérite une analyse de détail. Les passages concernés sont les suivants :

Lev 16,8

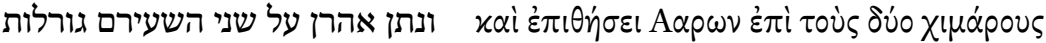

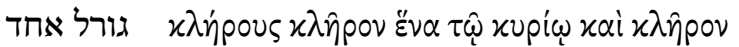

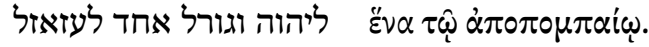

Et Aaron jettera des sorts sur les deux boucs, un sort pour Yhwh et un sort pour Azazel

Lev 16,10

והשעיר אשר עלה עליו הגורל לעזאזל יעמד חי לפני יהוה לכפר עליו

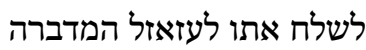

Le bouc sur lequel est tombé le sort pour Azazel sera placé, vivant, devant Yhwh pour faire l'expiation sur lui, pour l'envoyer à Azazel dans le désert.

Lev 16,26

והמשלח את השעיר לעזאזל יכבס

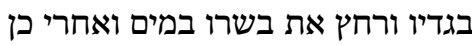
יבוא אל המחנה

Et celui qui a envoyé le bouc pour Azazel lavera ses vêtements et lavera son corps dans l'eau et, après cela, il viendra dans le campement.
Et Aaron placera sur les deux boucs des sorts, un sort pour le Seigneur et un sort pour l'éliminateur/celui qui part.

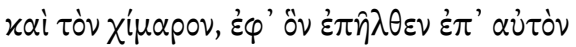

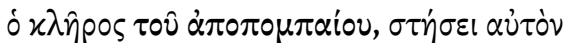

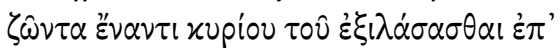

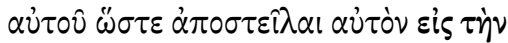

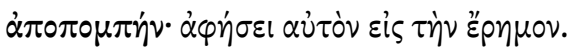

Et le bouc sur lequel est tombé le sort de l'éliminateur/celui qui part, il (Aaron) le placera vivant devant (le) Seigneur pour que soit faite l'expiation sur lui, de manière à le renvoyer pour éliminer le mal, il le lâchera dans le désert.

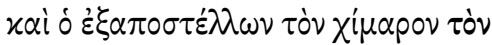

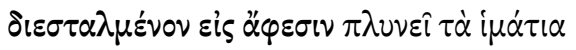

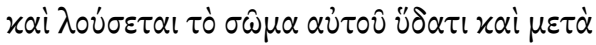

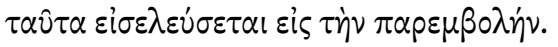

Et celui qui aura chassé le bouc qui a été séparé pour être lâché rincera ses vêtements et lavera son corps dans l'eau, et après cela il entrera dans le campement. 
Lors des trois premières occurrences, le traducteur recourt au mot $\dot{\alpha} \pi 0 \pi \circ \mu \pi \dot{\eta}$ et à ses dérivés. $L a \breve{a} z z \bar{a} z \bar{e} l$, «pour Azazel » en Lev 16,8 est rendu par l'adjectif substantivé $\tau \hat{\omega} \alpha \dot{\alpha} \pi \circ \pi \circ \mu \pi \alpha i \omega$. C'est ici la première fois que cet adjectif est attesté en grec, ce qui lui vaut d'être considéré comme un néologisme de la $\mathrm{LXX}^{70}$. Toutefois, ce terme ne semble pas être une innovation du traducteur car, bien que rarement, il est cité par des lexicographes et des savants alexandrins. Puisque la compréhension de l'ả $\pi 0 \pi \circ \mu \pi \alpha i ̂ \varsigma$ du verset 8 ne fait pas l'objet d'un consensus parmi les chercheurs, il convient de réfléchir à partir du mot $\alpha$ $\pi 0 \pi 0-$ $\mu \pi \eta$ qui est mieux attesté.

'A $\pi \circ \pi \circ \mu \pi \dot{\eta}$ a deux principaux domaines d'usages: un domaine juridique avec le sens de « divorce», bien attesté dans les papyri gréco-romains ${ }^{71}$, et un domaine religieux où il signifie « détournement» ou « élimination du mal », « conjuration», « expiation » ${ }^{72}$. Cette signification est déjà attestée à l'époque classique, notamment dans un passage d'Isocrate qui décrit les différentes manières de détourner les puissances mauvaises ${ }^{73}$. Elle est encore présente à l'époque impériale, à la fois dans la langue littéraire (Marc Aurèle parle de l'à $\pi \circ \pi \circ \mu \pi \eta \dot{n}$, à savoir la chasse aux démons ${ }^{74}$ ) et épigraphique (une gemme magique retrouvée sur la mer Noire mentionne l' $\alpha \pi \circ \pi \circ \mu \pi \dot{\eta}$ du $\left.\varphi \alpha \rho \mu \alpha x o ́ \varsigma^{75}\right)$. Le contexte de Lev 16 indique clairement qu'en Lev 16,1ob le terme est employé dans son sens religieux de «détournement», «élimination», « départ» lors du rituel. Ce choix pourrait être justifié, comme le considère la majorité des chercheurs, par une lecture étymologique de $\breve{a} z \bar{a} z \bar{e} l$ comme composé de 'z, «bouc», et 'zl, «partir», « disparaître».

Ladjectif $\dot{\alpha} \pi \circ \pi \circ \mu \pi \alpha \hat{\imath} \circ$, choisi par le traducteur pour rendre le nom d'Azazel aux versets 8 et 1oa, désigne une fonction qui a donc à voir avec l'à $\pi \circ \pi \circ \mu \pi \eta^{\prime}$, l'action de détourner. La majorité des traducteurs modernes comprend l'expression au passif, comme signifiant «à celui qui doit être envoyé, ou éliminé » ${ }^{76}$. Pour être compréhensible, une telle traduction impliquerait que $\delta \dot{\alpha} \pi \circ \pi \circ \mu \pi \alpha \hat{i} \circ \varsigma$ se réfère au bouc envoyé au désert et nommé au verset précédent, et qui serait sous-entendu au verset 8 . C'est effectivement ainsi que l'expliciteront les

\footnotetext{
$70 \quad$ Harlé et Pralon 1988, p. 79; Wevers 1997, p. 244.

71 Voir, par exemple, P.Fay 22, 21 ; PSI 1 36a, 16 ; P.Mich 5 340 Ro, 53 ; P. Ryl.Gr. 2 154, 29 ; CPR 1 236,8 ; BGU 1252,8 .

72 La signification rhétorique de « discours d'adieu » est, en revanche, rare et tardive (voir par exemple Men. Rh., p. 333 Spengel).

73 Isoc. 5, 117 : pour la discussion de ce passage voir p. 53-54. Voir aussi Luc., Philops. 9.

74 M. Ant. $1,6,3$.

$75 S E G$ 6o.817 (= 29. 731, l. 15); le terme apparaît, en outre, en $S E G$ 30.86 (= 21. 507) parmi des documents athéniens sur les artistes et les travailleurs du théâtre de Dionysos, dans un passage où le texte est très lacunaire.

76 Ainsi Büchner, «the one to be sent off» (NETS); Vahrenhorst 2011, «Weggeschickten » (LXX. Deutsch), et également Wevers 1997, p. 244-245.
} 
réviseurs anciens. Toutefois, le bouc n'est pas mentionné dans le texte grec de Lev 16,8b. En revanche, l'explication la plus simple et vraisemblable serait

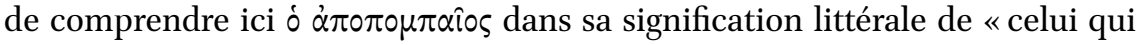
éloigne», « celui qui élimine» («l'éliminateur»), comme le suggèrent Paul Harlé et Didier Pralon ${ }^{77}$; il pourrait, encore, avoir la valeur active, mais intransitive, de « celui qui part». Dans les deux cas, l'expression fait référence à une puissance qui est encore mise en parallèle avec Yahweh ( $\tau \hat{\omega}$ xupi $\omega)$ ). Une brève enquête sur l'adjectif pourrait appuyer cette hypothèse.

Le suffixe $-\alpha 10 \varsigma$ dont l'adjectif est composé est très fréquent dans la forma-

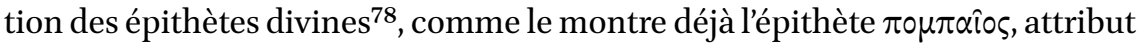
d'Hermès signifiant «celui qui conduit, qui transporte» (sous-entendu, les

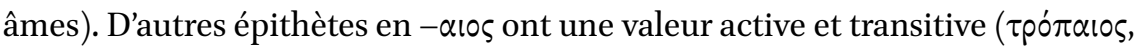
$\sigma \tau p \circ \varphi \alpha \hat{i} \circ \varsigma, \pi \alpha \nu \circ \mu \varphi \alpha \hat{i} \varsigma$, etc.). De plus, d'après les lexicographes alexandrins, les $\alpha \pi \circ \pi \circ \mu \pi \alpha \hat{0} \circ$ étaient une catégorie de divinités auxquelles on sacrifiait pendant des jours spécifiques de l'année ${ }^{79}$. Le lexique d'Harpocration mentionne également cette catégorie de divinités, en citant comme source Apollodore d'Athènes qui travaillait à la bibliothèque d'Alexandrie au $\mathrm{II}^{\mathrm{e}}$ siècle avant notre ère ${ }^{80}$. La Souda démontre en outre l'équivalence entre $\alpha \pi 0 \pi 0 \mu \pi \eta \dot{~ e t ~}$ $\alpha \pi 0 \tau \rho \circ \pi \dot{\eta}$, en expliquant le premier comme un synonyme moins courant $\mathrm{du}$ deuxième ${ }^{81}$. Cette équivalence laisse entendre que les dieux $\alpha \pi \circ \pi \circ \mu \pi \alpha \hat{i} \circ \mathrm{l}$ agissent de la même manière que les puissances désignées comme $\alpha \pi 0 \tau p \circ \pi \alpha \hat{\imath}$, « apotropaïques », dont la première fonction est d'écarter, d'éloigner le mal ${ }^{82}$. D'autres auteurs anciens attribuent à $\alpha \pi 0 \pi \circ \mu \pi \alpha \hat{\imath}$ ऽ une valeur intransitive de « partir», «s'écarter» : c'est le cas de Philon qui donne une interprétation allégorique du passage ${ }^{83}$. La valeur intransitive de « partir» est également possible pour Lev 16,8 et correspondrait bien à une lecture étymologique de la racine hébraïque ' $z l$.

À la lumière du domaine sémantique recouvert par $\alpha \pi 0 \pi \circ \mu \pi \dot{\eta}$ et $\alpha$ jo$\pi \circ \mu \pi \alpha i o s$, il semble donc possible d'affirmer que dans la LXX de Lev 16,8b

$77 \quad$ Harlé et Pralon 1988, p. 152.

78 Chantraine 1968, p. 46-47.

79 D'après Hésychius, les $\dot{\alpha} \pi \circ \pi \circ \mu \pi \alpha i$ sont les jours pendant lesquels on sacrifiait aux dieux

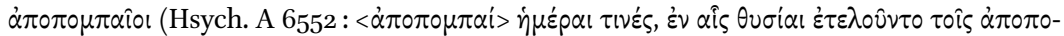
$\mu \pi \alpha$ ioıs $\theta$ вoîs).

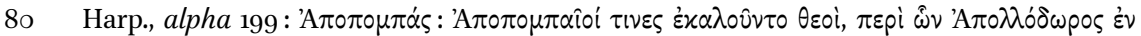

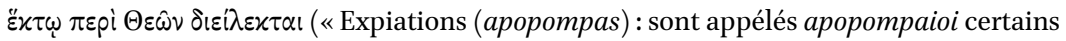
dieux dont Apollodore discute dans son sixième livre 'sur les dieux' »).

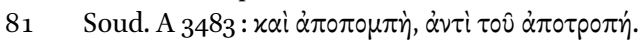

$82 \quad$ Voir supra, p. $56-58$.

83 Philon (Her. 179), d'après lequel le deuxième bouc représente l'esprit qui « s'est écarté loin de la sagesse » ; mais voir également Hsych., A $655^{2}:<\dot{\alpha} \pi 0 \pi 0 \mu \pi \alpha \hat{i} \circ \varsigma>\circ \dot{\alpha} \pi \circ \sigma \tau p \alpha \varphi \varepsilon i \zeta$. 
l'expression $\tau \hat{\omega} \hat{\alpha} \pi \circ \pi \circ \mu \pi \alpha i \omega$ ne se réfère pas au bouc qui doit être éliminé, mais porte une valeur active ${ }^{84}$ et fait, ainsi, référence à une puissance clairement en relation avec des rituels de détournement qui a la capacité d'écarter ou d'éloigner, même si elle n'est pas nommée.

Les raisons qui motivent ce choix de traduction restent difficiles à établir. Une volonté délibérée d'effacer le nom d'Azazel du rituel ne peut pas être complètement écartée ${ }^{85}$. Si le traducteur du Lévitique avait connaissance des traditions du Second Temple présentant Azazel comme un ange déchu, il serait alors compréhensible qu'il ait voulu ici éviter toute référence à cette entité. En outre, cette démarche serait en accord avec un apparent faible intérêt de la LXX pour les spéculations sur les noms angéliques ${ }^{86}$. Nous sommes, toutefois, dépourvus de données sur la diffusion effective du mythe des Veilleurs en Égypte au III $^{\mathrm{e}}$ siècle avant notre ère, et notamment dans la forme qui contient une référence à Azazel, car les sources dont nous disposons sont toutes plus tardives ${ }^{87}$. Il est donc possible que les traducteurs du Pentateuque ignorassent tout simplement ces traditions. La traduction par $\dot{\delta} \dot{\alpha} \pi \circ \pi \circ \mu \pi \alpha \hat{i} \circ \varsigma$ pourrait alors dériver de la perte de compréhension du mot hébreu Azazel ${ }^{88}$. Cette option serait compatible avec la lecture étymologique du mot, une stratégie souvent employée pour contourner une difficulté de compréhension.

À cet égard, Harlé et Pralon s'interrogent sur les motivations qui ont poussé le traducteur à écarter dans ce contexte la série $\dot{\alpha} \pi \circ \tau \rho \varepsilon \dot{\pi} \omega / \dot{\alpha} \pi 0 \tau \rho \circ \pi \dot{\eta} / \dot{\alpha} \pi 0 \tau \rho \delta_{-}$ $\pi \alpha 10 \varsigma$, pourtant courante en grec classique, et à lui préférer des formations liées à $\alpha \pi 0 \pi \varepsilon \dot{\varepsilon} \mu \omega$. D'après eux, cette démarche serait motivée par la volonté d'éviter un langage dont la connotation aurait été ostensiblement attribuée au contexte cultuel grec ${ }^{89}$. Une telle hypothèse a été rejetée par Daniel Stökl Ben

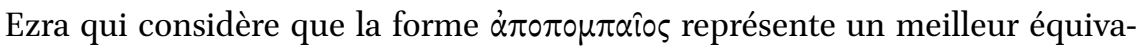
lent pour la forme Piel de la racine šl $h^{90}$. Je crois que les remarques de Stökl Ben Ezra sont bien fondées. Il faut effectivement remarquer que la famille

84 Ainsi également Lust, Eynikel, Hauspie, 2003, s.v. ; Muraoka, 2009, s.v. Voir également la discussion en Riedweg 2012, p. 454-459.

85 Ainsi Vahrenhorst 2011, p. 383 .

86 Les seuls noms d'anges dans la LXX sont Gabriel et Michaël dans le livre de Daniel, ce qui pourrait être expliqué par le rôle de premier rang joué par ces figures dans l'apocalyptique ; Raphaël est encore nommé dans le livre de Tobit. Voir, à ce sujet, l'étude de Cécile Dogniez dans Dogniez et Scopello 2006, p. 190-192, et infra, § 8.1.1.

87 Philon ne mentionne pas les noms des anges lorsqu'il traite du mythe des géants ; la section des Oracles Sibyllins qui contient une référence à d'Azazel est considérée d'origine chrétienne et donc plus tardive.

88 Ainsi Wevers 1997, p. 244.

89 Harlé et Pralon 1988, p. 151-152.

9o Stökl Ben Ezra 2003, p. 103-104. 


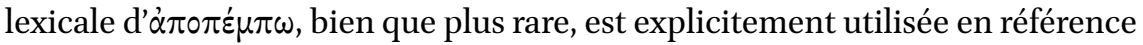
à des rituels de détournement et d'expulsion. Outre les sources déjà mentionnées, un passage du discours Contre Andocide, traditionnellement attribué à Lysias mais dont la paternité est douteuse, invoque la nécessité de purifier la ville en se libérant de ce personnage impie qu'est Andocide, alors comparé

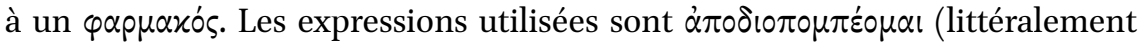

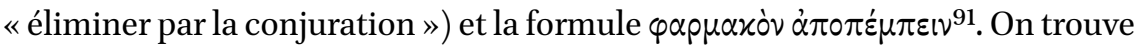

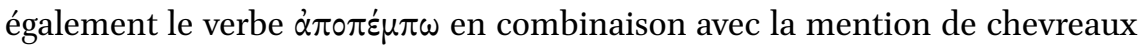
sauvages en contexte rituel dans un fragment des Aitia de Callimaque où il est question d'écarter une attaque de maladie, vraisemblablement épilepsie, qui saisit la jeune Cydippé ${ }^{92}$. À la lumière de cette documentation, il me semble donc que le souci du traducteur en Lev 16 fut de choisir une formulation capable de rendre compte, de la manière la plus précise possible, de la fonction attribuée à l'envoi du bouc dans le rituel. Dans ce but, il n'hésita pas à puiser dans les traditions rituelles grecques de conjuration où il choisit les expressions qui lui paraissaient les plus adéquates à son contexte, tout en étant respectueuses de l'hébreu subjacent ${ }^{93}$.

En revanche, il paraît indéniable que le choix du traducteur de garder la fonction d'une puissance divine au verset 8 lui posa quelques problèmes dans les versets suivants, vraisemblablement car il n'était pas en mesure d'identifier cette puissance. Il cherche alors à produire un texte qui ait du sense en grec en se détachant du principe isomorphique, d'après lequel à chaque élément du texte hébreu correspond un équivalent en grec. En effet, il apporte une série de changements par rapport au texte source, dont des modifications dans la lecture du nom d'Azazel. Au verset 10a, le parallélisme entre Yhwh/Azazel présent dans le тм est perdu car $\delta \dot{\alpha} \pi \circ \pi \circ \mu \pi \alpha \hat{o} \varsigma$ est mis au génitif au lieu du datif que le texte hébreu induirait. L'expression devient donc ambiguë car elle pourrait être effectivement comprise comme «le sort de celui qui part», en référence au bouc. Dans le même verset (1ob), à la deuxième occurrence du nom Azazel, le traducteur varie en utilisant le substantif $\dot{\alpha} \pi \circ \pi \circ \mu \pi \eta \dot{\eta}$ : «pour l'élimination (du mal)». Ce choix l'oblige à introduire un nouveau verbe suivi d'un objet

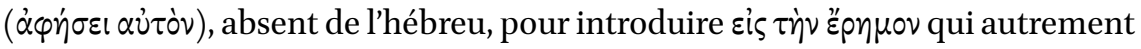

\footnotetext{
$91 \quad$ Ps.-Lys. 6, 53.

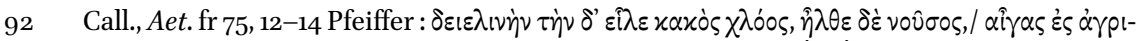

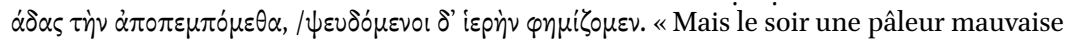
la saisit et survint une maladie, que nous chassons par des chevreaux sauvages et que nous appelons faussement 'sacrée' ».

93 Voir, également, Stökl Ben Ezra (2003, 102-106, et surtout p. 106) qui souligne le fait que le traducteur rend le texte compréhensible pour le public grec, à la fois juif et non juif.
} 
n'aurait pas de sens ${ }^{94}$. Enfin, au verset 26 la'ăzzà $z \bar{e} l$ est encore rendu par l'ex-

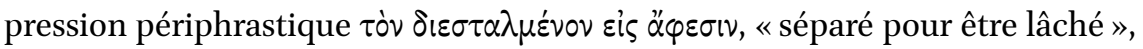

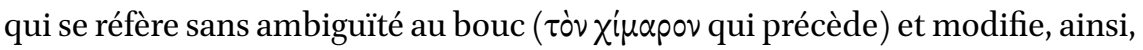
le sens du passage par rapport à l'hébreu. On a donc l'impression que le traducteur, mal à l'aise avec son premier choix de traduction, élimine la référence à une puissance divine protagoniste du rite d'élimination, tout en préservant en même temps l'accent sur la fonction du rituel.

Il mérite également d'être relevé que les réviseurs, au lieu de rétablir la signification littérale du mot hébreu selon leur démarche habituelle, adoptent pour la majorité la lecture étymologique ' $z+{ }^{\prime} z$ l et qu'ils interprètent le passage

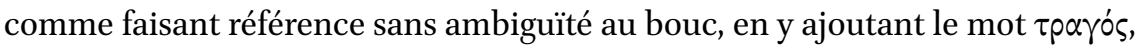
absent de la LXX (voir tableau $12 a$ en Appendice). Symmaque traduit la ăazzāzēl

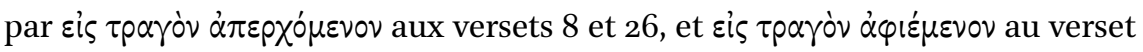
10. La tradition indirecte d'Aquila, rapportée par Théodoret, propose une ver-

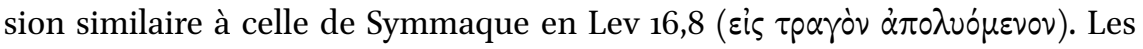
codices, en revanche, lisent $\varepsilon i \zeta \varsigma \varepsilon x p \alpha \tau \alpha i \omega ́ \mu \varepsilon v o v$, neutre dérivé d'une lecture de la racine ' $z$ qui indique vraisemblablement un lieu fortifié. Cette lecture attesterait donc que l'interprétation d'Azazel comme nom de lieu était déjà connue par Aquila ${ }^{95}$. La forme $A \zeta \alpha \zeta \eta \lambda$ apparaît presque exclusivement sur des témoignages minoritaires ou secondaires. Elle est néanmoins attestée sur une majus-

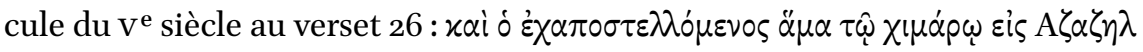
(« et celui qui est envoyé avec le bouc vers Azazel») ${ }^{96}$. D'après John Wevers cette lecture pourrait remonter à Aquila; toutefois, la formulation dans son ensemble ne permet pas d'exclure que Azazel soit ici encore compris comme un nom de lieu. La référence à Azazel est également absente chez Philon dans les différents passages où il discute la signification du rituel de conjuration, ainsi que chez Flavius Josèphe ${ }^{97}$.

En conclusion, la vision selon laquelle la LXx évacue complètement la présence d'une entité démoniaque dans le rituel de Lev 16 serait à nuancer. La référence à une puissance associée au rite de conjuration est encore claire pour le traducteur, surtout au versets 8 et 1oa. Toutefois, le rôle de cette puissance n'est pas totalement clair: a-t-elle pour fonction d'écarter les fautes («l'éliminateur », selon la valeur transitive d' $\alpha \dot{\pi} 0 \pi 0 \mu \pi \alpha \hat{\imath} \circ$ ) ou doit-elle être mise à l'écart avec les fautes du peuple (dans ce cas $\dot{\alpha} \alpha \circ \pi \circ \mu \pi \alpha \hat{\imath} \circ$ క serait « celui qui part»,

94 Parmi les autres différences entre hébreu et grec dans ce verset, on remarque que dans le texte grec Aaron est le sujet de l'action, à savoir celui qui «place» le bouc, alors qu'en hébreu le verbe est à la forme passive et que le sujet n'est pas spécifié.

95 Wevers 1986, ad loc. (apparatus I-II); Field 1875, p. 194.

96 Il s'agit d'une note hexaplaire (Cod. $\mathrm{x}$ en Field $=\mathrm{M}$ en Wevers).

97 Philo, Leg. 2, $5^{2} ;$ Her. 179, 187 ; Poster. 70-72 ; Plant. 61; Jos., AJ 3, 241. 
« qui disparaît ») ? Dans les deux cas, le traducteur semble mal à l'aise avec son choix de traduction et tend progressivement à éliminer cette référence.

En résumant, la tradition qui élimine toute référence à Azazel en traduisant le mot hébreu en lien avec le bouc envoyé au désert a effectivement ses origines dans la LXX, notamment en Lev 16,26. Elle est ensuite prolongée dans d'autres traditions grecques par les réviseurs ainsi que par Philon et Josèphe qui ne font plus mention d'Azazel lorsqu'ils discutent ce rituel. De manière générale, l'interprétation de la Lxx est à contre-courant d'autres traditions du Second Temple qui attestent, voire amplifient, le caractère « démoniaque» d'Azazel. S'il reste possible que l'Azazel du mythe des Veilleurs fût encore inconnu en Égypte à l'époque de la traduction du Pentateuque, cela est beaucoup plus difficile à imaginer dans le contexte de travail des réviseurs, à savoir la Palestine d'époque romaine.

\subsection{Les transformations de Lilith}

La présence de Lilith dans la Bible hébraïque peut être considérée à certains égards comme un cas de figure comparable à celui d'Azazel. Comme Azazel, Lilith n'est nommée qu'à un endroit isolé, dans le livre d'Isaïe, et ne fait pas l'objet d'une traduction littérale dans la LXx, bien que les procédés mis en œuvre par le traducteur d'Isaïe soient très différents de ceux adoptés par le traducteur du Lévitique. Comme Azazel, la figure de Lilith fait l'objet d'un développement important dans le judaïsme ancien. Quelques ouvrages récents ont essayé de nuancer la nature démoniaque de Lilith dans la Bible hébraïque, en se fondant sur la pauvreté des indications données par le texte biblique. Toutefois, le contexte plus large de la démonologie levantine et, de manière plus générale, proche-orientale, amène à rejeter avec conviction cette proposition.

Tout d'abord, la présence d'une famille de démons lilu/lilitu/ardat lili est bien attestée dans le recueil d'incantations Utukku Lemnutu et remonte au démon sumérien lil-. Il s'agit d'entités liées aux tempêtes et aux vents, souvent nommées en triade, qui peuvent prendre la forme d'apparitions ou de fantômes habitant les villes abandonnées et les lieux désertiques ${ }^{98}$. Dans les textes accadiens lilu, lilitu et ardat lili sont à comprendre comme des variantes masculines et féminines d'une même typologie de démon plutôt que comme des noms «propres »: de manière générale, ces puissances sont d'ailleurs mal distinguées les unes des autres ${ }^{99}$. Une incantation contre Lamaštu dit qu'elle

98 Ils sont systématiquement nommés en série de deux ou trois dans les Utukku Lemnutu: 1 (add.), $13 ; 2,65 ; 6,60 ; 9,87 ; 10,32-34 ; 13-15,138,179$.

99 Farber 1987. L'entité la mieux caractérisée semble être ardat lili alors que lilitu n’apparaît quasiment jamais comme nom isolé. Le seul récit mythologique où la démone lil-joue un 
«vole autour» comme un lilu: le fait qu'elles soient représentées comme volantes permet de les associer à des oiseaux, une caractéristique qui est partagée par la plupart des démons proche-orientaux ${ }^{100}$. La présence de démons féminins volants et dangereux semble également confirmée sur l'amulette d'Arslan Tash: le terme employé est ici différent (' $p$ '), mais indique néanmoins qu'une typologie similaire est connue et familière au Levant ${ }^{101}$. Dans les sources accadiennes plus récentes, un aspect de sexualité agressive et déréglée est associé à ce groupe, ce qui est également un trait courant de la représentation des démons féminins dans l'Antiquité, comme le montre l'exemple bien documenté de Lamaštu. Ardat lili apparaît également comme tueuse et ravisseuse de nouveau-nés. Il faut toutefois observer que, dans les textes accadiens, le démon «femelle » ardat lili autant que le démon «mâle» lilu représentent un danger pour les nouveau-nés : ardat lili n'est en ce sens qu'un pendant féminin du lilu, lui aussi conçu comme un démon de la luxure, caractérisé par une sexualité déréglée. Les textes médicaux, par exemple, préviennent contre le risque qu'un lilu s'approche du bébé et des femmes enceintes ${ }^{102}$.

Dans les traditions du judaïsme ancien, Lilith est une démone de premier rang qui a plusieurs facettes. Sa nature sexuellement dangereuse est mise en avant dans le Talmud ${ }^{103}$. Dans les Targums un démon nocturne est nommé

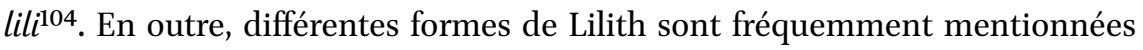
sur les amulettes, les bols et les incantations araméennes de Babylone et Nippur. Là, elles sont qualifiées au singulier, au pluriel et dans quelques cas comme des «liliths mâles et femelles » ${ }^{105}$. Sa physionomie apparait ici bien plus complexe que sa caractérisation dans le texte biblique. La nature de Lilith en tant que démone de la sexualité est déjà connue par les réviseurs de la LXX :

rôle se trouve dans la version sumérienne de l'épopée de Gilgamesh (pour une analyse, voir Ribichini 1987).

100 Lam. I 114, Farber 2014, p. 155, 211. Voir également la «tablette de l'ardat lili» publiée par Lackenbacher 1971, col. I, ll. 6-8, p. 140.

101 Amulette 1, l. 19, voir Pardee 1998, p. 19; supra, p. 29. En revanche, la vieille lecture de $l$ comme Lilith à la ligne 20 est désormais à rejeter pour des raisons épigraphiques (Pardee 1998, p. 25-26).

102 Voir CAD 9, p. 190 c; Farber 1987, p. 23.

103 b.Erub. 11b; b.Nid. 24b; b.Šabb. 151b ; b.B.Bat. 73b ; b.Pesah 112b. Une démone avec des caractéristiques similaires, mais portant un nom différent, mentionnée dans le Testament de Salomon (13, 1-4) est souvent rapproché de Lilith. Dans les traditions juives médiévales représentées par l'Alphabet de Ben Sira et le Zohar, Lilith devient la première épouse d'Adam. Voir, pour un premier aperçu, Scholem 2007; Hutter 1999 b, p. 520-521. Une bibliographie assez abondante est donnée par Blair 2009, p. 26-29.

104 Targum Ps-Jo. en Deut 32,24 et Num 6, 24.

105 Par exemple Naveh et Shaked 1998, B 1, 3,6, 11 ; 5,$6 ; 12 \mathrm{a}$, XI ; 13, 1, 7, 22 ; 8, 1. Sur la complexité des graphies du nom de Lilith dans les incantations araméennes, voir Müller-Kessler 2001. 
en Is 34,14, Symmaque (suivi ensuite par Jérôme) suggère de rendre l'hébreu Lilith par le grec $\lambda \alpha \dot{\mu} \mu \alpha$, (lat. Lamia ${ }^{106}$. Non seulement cette traduction rend compte de son caractère démoniaque, mais elle permet également de l'associer aux figures féminines des traditions grecques et romaines liées à l'épouvante et à la sexualité incontrôlée.

Lattestation du nom de Lilith dans les documents qumrâniens est un élément dont il faut également tenir compte. Son nom apparaît dans le rouleau d'Isaïe mais aussi dans un texte connu comme les « Chants du Sage », préservé sur deux rouleaux (4Q510 et $\left.5^{11}\right)^{107}$. Il s'agit de l'un des textes les plus éloquents pour la démonologie qumrânienne où Lilith est nommée parmi une liste des démons que le chef de la communauté se charge d'exorciser. Ici la forme plurielle lylywt $t^{108}$ fait encore penser à une typologie ou à un groupe de démons qui partagent des caractères similaires, sur le modèle des démons lil-accadiens.

Par conséquent, étant donné l'importance de l'arrière-plan proche-oriental des démons du type lil-, couplé à la présence de Lilith dès les traditions du Second Temple, dans la tradition juive ancienne ainsi que dans une partie de la tradition grecque où elle est toujours comprise comme une démone, il serait assez surprenant qu'elle cesse d'être considérée comme telle exclusivement lors de son apparition dans la Bible hébraïque. Effectivement, le rédacteur d'Isaïe 34 semble bien connaitre les traditions sur Lilith, et le fait qu'elle soit nommée dans un oracle sur Babylone rappelle le lieu d'origine de ce démon. Il faut, toutefois, admettre que Lilith est présentée dans la Bible hébraïque de manière singulière. En effet, deux aspects fondamentaux de cette démone, à savoir sa nature agressive et ses connotations sexuelles, sont absents du texte biblique. En revanche, sa nature animalière - possiblement d'oiseau - est mise en avant, de même que le fait qu'elle habite parmi les ruines. Il s'agit là de caractéristiques propres à la représentation de plusieurs typologies de démons, y compris les démons lil-. Quoiqu'il en soit, la fonction de Lilith dans ce passage doit être inscrite et comprise dans le contexte littéraire d'Isaïe 34,

106 Ziegler 1939 ad loc., apparatus II.

107 Édité par Maurice Baillet (1982, DJD 7, p. 215-262). La restitution de lilith proposée par Émile Puech (2000, p. 164) en 11Q11 6 l. 1 n'est pas appuyée par des arguments solides et est donc à rejeter. La possibilité que la femme décrite en 4 Q184 ait des connotations démoniaques, comme suggéré par Baumgarten (1991), ne permet pas pour autant de bel et bien y voir une Lilith. Comme le souligne Matthew Goff (2016), la femme séductrice de 4Q184 doit être vraisemblablement interprétée à lumière des traditions sur la « dame folie» attestées dans le livre des Proverbes. Pour une mise au point sur les différentes interprétations de ce texte et une critique de l'hypothèse de Baumgarten, voir Goff 2016, surtout p. $67-68$ et $71-73$.

1084 Q51o fr.1, l. 5 ; 4Q511 fr. 10, l. 5 (Baillet 1982, DJD 7, p. 220-221, 226-27) et infra, § 5.4. 
qui sera discuté en détail par la suite. Mais dans la LXx d'Isaïe 34 il n’y a manifestement pas de traces «littérales» de Lilith, dont le nom est rendu par ỏvo-

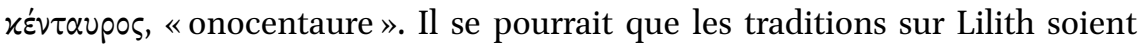
inconnues à cette époque en Égypte, ce qui expliquerait aussi son absence des papyri magiques gréco-romains, au contraire de ce qui arrive dans les textes magiques araméens d'origine palestinienne où elle joue un rôle important.

Si Lilith et Azazel partagent le fait d'être, en quelque sorte, transformés dans la LXX, il faut néanmoins noter les différences de traitement que leur réservent les divers traducteurs. Dans la Lxx d'Isaïe 34, le passage dans son ensemble conserve sa connotation « démoniaque »; connotation notamment amplifiée par toute une série d'équivalents choisis dans le texte. Ce n'est d'ailleurs pas un hasard si cela correspond précisément à l'un des contextes où le mot $\delta \alpha \mu$ óviov fait son apparition. En effet, la transformation de Lilith en onocentaure et la présence des $\delta \alpha \mu o ́ v i \alpha$ dans la LXX d'Isaïe répond à une logique précise du traducteur qui s'inscrit dans un scénario du démoniaque spécifique à la LXX, lequel méritera une analyse plus détaillée.

Cette enquête sur les équivalences grecques choisies pour Azazel et Lilith dans la LXX amène donc à conclure que l'idée selon laquelle le potentiel démoniaque associé à ces créatures est complètement perdu dans la traduction grecque de la Bible hébraïque est à réviser. Même si le référent précis derrière ces noms peut avoir échappé aux traducteurs, les procédés mis en œuvre dans les deux cas montrent que ce potentiel était bien connu, faisant l'objet de nouvelles transformations. En revanche, cela ne semble pas avoir été le cas pour le démon « caché » en Gen 4.

\section{Un « démon de la porte »? (Gen 4,7$)$}

Lorsque l'on s'interroge sur la présence ou sur l'absence des démons dans la Bible, on ne peut pas omettre de discuter, au moins brièvement, l'obscur passage de Gen 4,7, qui a fait couler beaucoup d'encre en raison de ses difficultés textuelles. Le contexte, bien connu, est celui du conflit entre Caïn et Abel et, notamment, de la frustration de Caïn face à la préférence de Yhwh pour les offrandes de son frère, ce qui aura des conséquences funestes. Lorsque Caïn a «le visage abattu » (versets $5^{-6}$ ), Yhwh lui adresse la parole:

הלוא אם תיטיב שאת ואם לא תיטיב לפתח חטאת רבץ ואליך תשוקתו ואתה לאיב

תמשל בו לפות

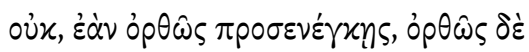

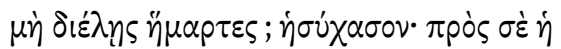

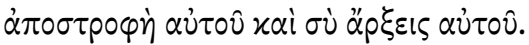


Si tu agis bien, soulever (ne le soulèveras tu pas -scil. ton visage)? Mais si tu n'agis pas bien, à la porte péché est tapi, et il te désire. Mais toi, domine-le.
Si tu présentes correctement (scil.l'offrande) mais sans diviser correctement, n'as-tu pas commis une faute? Reste calme, son inclination est vers toi, et tu le commanderas.

Le texte hébreu présente une série de difficultés qui ont amené certains chercheurs à considérer ce passage comme «intraduisible »109. Comme il m'est ici impossible d'en examiner tous les problèmes textuels, je me limiterai à ceux qui sont pertinents pour mon argument. Outre la difficulté de compréhension posée par l'infinitif construit śa'êt, la traduction ici proposée, comme la plupart des traductions modernes, présente un paradoxe, à savoir que hațtâ̄t « " péché » est un substantif féminin, mais est pourtant suivi par une série de concordances au masculin. De plus, l'action se déroulant dans les champs, la signification de l'entrée ou porte (lappetah) n'est pas évidente. En outre, la position de lappetah («devant la porte») qui précède hatțăt est inhabituelle: selon la logique de la phrase nous l'attendrions plutôt après robeș. Pour éclairer ce passage, plusieurs chercheurs ont suivi et suivent encore une ancienne hypothèse de Hans Duhm ${ }^{110}$ qui voyait en $r b s ̦$ non un participe du verbe "s'attarder», «se reposer», «se coucher», tel qu'il apparaît ailleurs dans la Bible hébraïque, mais le nom d'un démon emprunté à l'accadien rabișu, bien connu par les textes incantatoires mésopotamiens. Le péché serait donc représenté comme un démon tapi à la porte, une sorte d'agent divin envoyé pour contrôler Caïn, et, par conséquent, les actions suivantes devraient être attribuées à ce démon ${ }^{111}$. Les liens intertextuels clairs entre Gen 4,7 et Gen 3,16, dont Gen 4,7c reprend explicitement le langage, ont amené certains à voir en $r b s ̦$ une réminiscence du serpent de Gen 3, notamment en raison du fait que la racine $r b s ̦$ dans la Bible hébraïque se rapporte souvent à des animaux ${ }^{112}$. Cette proposition demeure cependant trop spéculative et doit donc être à rejeter. Néanmoins, l'hypothèse d'un « démon de la porte » a connu un large succès, sans pourtant parvenir à résoudre tous les problèmes de ce passage ${ }^{113}$. En revanche, les solutions alternatives qui ont été avancées impliquent un certain degré de remaniement du

\footnotetext{
109 Ainsi Skinner 1930, p. 107 ; Westermann 1972, p. 407.

110 Duhm 1904, p. 8-14.

111 Pour différentes déclinaisons de cette hypothèse on peut voir, entre autres, Closen 1935, et parmi les études récentes, Gordon 2011; Schlimm 2012 ; Loiseau 2013; Kitz 2016. La bibliographie plus ancienne sur le sujet est donnée par Westermann 1972, p. 406.

112 Ainsi, récemment, Gordon 2011, mais déjà Duhm 1904, p. 10. Pour $r b s ̣$ comme verbe qui peut servir à décrire le comportement animal, voir Waschke 2004 ; Kitz 2016, p. 252.

113 Voir, en ce sens, les remarques de Barré 1999, p. 683.
} 
texte. Récemment, C.L. Crouch a, à nouveau, proposé l'idée que hatțāt soit une glose insérée tardivement dans le texte ${ }^{114}$ : le grec révèle cependant que ce mot était déjà dans le texte au moment de la traduction. D'autres fournissent une interprétation exclusivement métaphorique et extrêmement élaborée du passage, dont la comprehension n'est pas tout à fait claire ${ }^{115}$.

L'interprétation de $r b s ̦$ comme nom de démon est donc souvent acceptée « faute de mieux » et elle paraît effectivement appuyée par les parallèles mésopotamiens. Non seulement l'action de se cacher dans les coins est propre aux démons, mais de manière générale le seuil est le lieu d'accès privilégié pour les attaques démoniaques. En outre, comme le relève justement Anne-Marie Kitz, l'idée du rabiṣu qui « s'attache » à l'homme est présente dans les sources accadiennes ${ }^{116}$ et la représentation d'un démon "guetteur» qui se cache ou qui rôde correspond bien à l'étymologie du mot ${ }^{117}$.

Quelques problèmes demeurent cependant. Cette solution oblige à comprendre la racine $r b s ̣$ dans un sens nominal qui n'est jamais attesté ni en hébreu ni dans d'autres langues ouest-sémitiques alors que la racine verbale est bien attestée. En outre, la représentation démoniaque du péché qui, caché dans un coin, cherche à séduire l'homme n'est attestée nulle part ailleurs dans la Bible où hatțăt est plutôt un terme connoté du point de vue cultuel : il indique une typologie sacrificielle précise en contexte rituel. Même quand ce terme a une signification générique de «faute», par exemple dans ses quelques autres occurrences dans la Genèse, il indique simplement une faute commise contre un membre de la communauté ${ }^{118}$. Dans la Bible hébraïque, on ne saurait trouver la moindre trace d'une «personnalisation» de la faute, ni d'une conception du péché représenté comme une puissance autonome ou comme un agent divin ${ }^{119}$. Une interprétation démonologique n'est pas davantage attestée dans les traditions postibliques, et ce en dépit du fait que le motif de l'envie

114 Crouch 2011 ; mais il s'agit d'une proposition qui avait déjà été avancée par Gunkel 1977, p. 43-44.

115 Bernd Janowski (2003, p. 150-151), suivi par Frey-Anthes (2007, p. 78) considère Abel comme étant le sujet de la phrase et donne une traduction dont la compréhension demeure assez compliquée : «und wenn du es nicht gut handelst, lagert er (scil. Abel) sich als (Öffnung >) Anlass zu einer Verfehlung ». Particulièrement complexe sur le plan syntactique et sémantique est également la proposition d'Ellen van Wolde (1991, p. 31-32): «there is at the door sin, (namely) being on the lurk? The desire to be on the lurk is for you, but you can master it ». Pour d'autres hypothèses, voir Erzberger 2011, p. 49-52.

116 Les sources sont examinées par Kitz 2016, p. 454.

117 Edzard et Wiggermann 1989, p. 449-455.

118 Gen 18,$20 ; 31,36 ; 5^{\circ}, 17$.

119 Pour un premier aperçu des usages de ḩațtāt dans la Bible Hébraïque, voir Koch 1980. 
préminent en cet épisode aurait pu offrir l'occasion pour un développement exégétique en cette direction.

Il se peut enfin que le passage soit corrompu. Le Pentateuque samaritain donne un texte différent dans la première partie du verset ${ }^{120}$, mais identique dans la deuxième qui fut également transmise sans variations significatives dans les versions anciennes. La traduction de la Lxx témoigne d'un texte consonantique proche du texte hébreu, exception faite de lpth, qui a été lu comme lnth, «diviser», dans le sens de «faire les parties pour le sacrifice». La traduction de tšw $q h$ par $\dot{\alpha} \pi \circ \sigma \tau \rho \circ \eta \dot{\eta}$ pourrait indiquer une compréhension de tšwqh comme tšs $b h$, «retour», mais cette lecture n'implique pas néces-

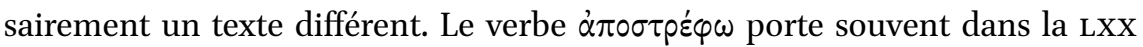
la signification de «revenir », «retourner vers le point de départ» (un sens,

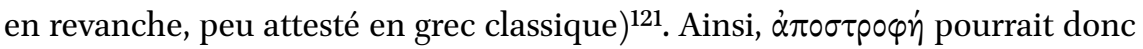
bien servir à rendre la notion d' « inclination », « destin inexorable », une signification qui est possible pour tšwqh dans ses quelques occurrences bibliques et qui est surtout très bien attestée à Qumrân ${ }^{122}$. Mais il reste impossible de reconstruire avec précision les étapes de l'histoire textuelle du verset.

En revanche, il est clair que l'interprétation de ce verset s'avérait déjà problématique pour le traducteur grec qui lui donne un sens différent par rapport à l'hébreu. Dans la première partie du passage, le traducteur rend explicite la nature de la faute de Caïn : il s'agit d'une erreur rituelle, qui prend la forme d'une mauvaise division des parties du sacrifice ${ }^{123}$. Hațtăt et $r b s ̣$ sont compris par le traducteur comme étant deux verbes, le deuxième étant lu à la forme impérative, rabaș. La deuxième moitié du verset traduit l'hébreu mot à mot, en aboutissant à un texte qui est encore partiellement énigmatique car le référent

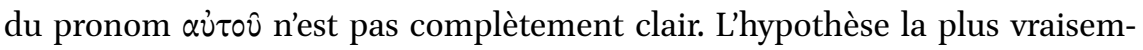
blable, à mon avis, est que le pronom fasse référence à Abel, et que l'hémistiche soit une anticipation du passage qui suit (Gen 4,8a), absent du тм mais présent dans toutes les versions anciennes : « et Caïn dit à Abel : allons dans la plaine».

En conclusion, dans le texte hébreu la possibilité de lire rbṣ comme le nom d'une entité démoniaque sur le modèle du rabișu mésopotamien ne peut pas être complètement exclue bien qu'elle ne parvienne pas à résoudre toutes les

\footnotetext{
הלוא אם תטיב לפתח חטאת רבץ ואליך תשוקתו ואתה תמשל בו 120.

121 Voir, par exemple, Gen 24,$5 ; 28,15$ et 21.

$1221 \mathrm{QS} \mathrm{11,} 22$; 1QM 15, 9-10; et 17, 4. Voir, déjà, à ce propos, les remarques de Closen 1935, p. 433. Récemment Loader 2014, p. 383. Anne-Françoise Loiseau (2016, p. 158-162) y voit une influence de l'araméen $m t w y$ ', « désir », «élan », qui, à mon avis, n'est pas nécessaire pour justifier ce choix de traduction.

123 Voir les remarques de Harl 1986, p. 114, reprises ensuite par Dorival 1996, p. 111.
} 
difficultés de compréhension du passage. On peut, certes, rejeter l'interprétation de $r b s ̣$ comme nom propre et attribuer à la racine $r b s ̣$ la signification verbale mieux attestée de «s'attarder» ou «se tapir», mais, même dans ce cas, la signification générale du passage ne subit pas de variation significative car le péché apparaît encore représenté comme un agent qui a pour fonction de surveiller ou de guetter Caïn. En l'état actuel du texte, cette lecture demeure la plus vraisemblable, notamment car aucune interprétation alternative ne semble pouvoir s'imposer davantage. On pourrait alors se demander si ce n'est en tant qu'agent divin que hatța $\vec{t}^{\prime}$ serait ici traité comme un nom masculin.

Quoi qu'il en soit, le traducteur grec, bien qu'en lisant en Gen 4,7 un texte très similaire au $\mathrm{TM}$, n'a gardé aucun souvenir de ce «démon de la porte». Cette disparition ne doit pas être imputée à des raisons d'ordre théologique, mais plutôt à la difficulté de déchiffrement du verset. L'arrière-plan démonologique du rabișu était probablement complètement étranger au traducteur, de même que la représentation du péché comme agent divin qui reste sans parallèle dans la Bible hébraïque. Ainsi, dans l'idée d'offrir une traduction harmonisante, le traducteur a vraisemblablement compris hatt $\bar{a}^{\prime} t$ comme une racine verbale permettant d'inscrire le verset dans le contexte immédiat du passage (Gen 4,3-5), soit celui cultuel des offrandes et sacrifices présentés par les deux frères.

Le sondage préliminaire des occurrences de $\delta \alpha i \mu \omega \nu / \delta \alpha \mu o_{v}$ iov et la discussion sur les démons qui semblent avoir «disparu» de la LXX permet déjà d'avancer une série d'observations d'ordre plus générale concernant l'arrière-plan culturel et idéologique des traducteurs, le pluralisme interne à la LXX ainsi que la place de cette dernière à l'intérieur du judaïsme ancien. Les exemples de $\delta \alpha \mu o ́ v i o v$ et de $\alpha \pi \circ \pi \circ \mu \pi \alpha i o \zeta$ montrent une continuité considérable avec le langage religieux grec, continuité qui est supérieure à ce que

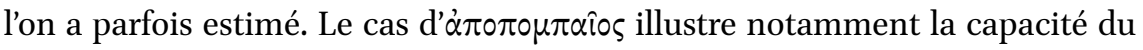
traducteur d'adapter le vocabulaire de la pratique rituelle grecque pour exprimer de manière soignée le sens du texte hébreu même dans des circonstances difficiles : à savoir lorsque le référent précis, c'est à dire Azazel, a été perdu. Au

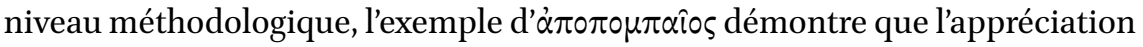
de l'habilité du traducteur peut et doit être mesurée sans se focaliser exclusivement sur les aspects « exceptionnels » des prétendus néologismes, qui sont d'ailleurs un phénomène fréquent en grec postclassique ${ }^{124}$, mais en prenant en compte le contexte plus large des familles lexicales $(\dot{\alpha} \pi \circ \pi \dot{\varepsilon} \mu \pi \omega / \alpha \dot{\alpha} \pi 0 \pi \circ \mu \pi \dot{\eta} /$

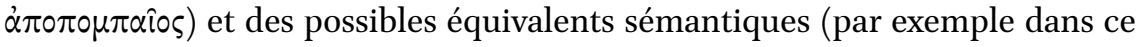

124 Pour l'identification et le traitement des néologismes dans la LXX voir les remarques méthodologiques fondamentales de Aitken 2013. 


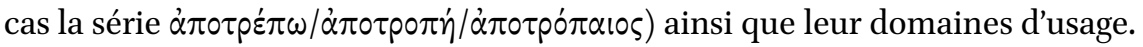
Par conséquent, le dossier traité dans ce chapitre suggère que l'importance des soucis théologiques sur le travail des traducteurs doit être redimensionnée. Les cas du « démon de la porte » disparu de Gen 4,7 confirme, certes, que le travail de traduction implique toujours un processus exégétique; néanmoins, un tel processus vise moins à masquer la présence d'entités démoniaques qui auraient été problématiques, quà restituer un passage autrement incompréhensible de manière adaptée à son contexte narratif. En outre, une telle pratique ne correspond pas nécessairement à l'invention personnelle du traducteur, mais est probablement en accord avec une exégèse courante de l'époque, où l'interprétation en sens «démonologique» de ce passage n'est jamais attestée. En revanche, les interprétations d'Azazel et Lilith, où la LXX est en contraste avec d'autres tendances exégétiques contemporaines, soulèvent la question du caractère distinctif du judaïsme alexandrin (et, de manière plus générale, égyptien), par rapport au judaïsme palestinien et babylonien. Dans les deux cas, la traduction grecque ouvre une fenêtre sur l'exégèse la plus ancienne de la Bible hébraïque à laquelle on n'aurait autrement aucun accès et qui mérite donc d'être mise en valeur. 